\title{
ARTICLE Intravenous formulation of Panax notoginseng root extract: human pharmacokinetics of ginsenosides and potential for perpetrating drug interactions
}

\author{
Salisa Pintusophon ${ }^{1,2}$, Wei Niu ${ }^{1}$, Xiao-na Duan ${ }^{1,2}$, Olajide E Olaleye ${ }^{1}$, Yu-hong Huang ${ }^{3}$, Feng-qing Wang ${ }^{1}$, Yan-fen Li ${ }^{3}$, \\ Jun-ling Yang ${ }^{1}$ and Chuan $\mathrm{Li}^{1,2}$
}

XueShuanTong, a lyophilized extract of Panax notoginseng roots (Sanqi) for intravenous administration, is extensively used as addon therapy in the treatment of ischemic heart and cerebrovascular diseases and comprises therapeutically active ginsenosides. Potential for XueShuanTong-drug interactions was determined; the investigation focused on cytochrome P450 (CYP)3A induction and organic anion-transporting polypeptide (OATP)1B inhibition. Ginsenosides considerably bioavailable for drug interactions were identified by dosing XueShuanTong in human subjects and their interaction-related pharmacokinetics were determined. The CYP3A induction potential was determined by repeatedly dosing XueShuanTong for 15 days in human subjects and by treating cryopreserved human hepatocytes with circulating ginsenosides; midazolam served as a probe substrate. Joint inhibition of OATP1B by XueShuanTong ginsenosides was assessed in vitro, and the data were processed using the Chou-Talalay method. Samples were analyzed by liquid chromatography/mass spectrometry. Ginsenosides $\mathrm{Rb}_{1}$, $\mathrm{Rd}$, and $\mathrm{Rg}$, and notoginsenoside $\mathrm{R}_{1}$ were the major circulating XueShuanTong compounds; their interaction-related pharmacokinetics comprised compound dosedependent levels of systemic exposure and, for ginsenosides $\mathrm{Rb}_{1}$ and $\mathrm{Rd}$, long terminal half-lives (32-57 and 58-307 h, respectively) and low unbound fractions in plasma $(0.8 \%-2.9 \%$ and $0.4 \%-3.0 \%$, respectively). Dosing XueShuanTong did not induce CYP3A. Based on the pharmacokinetics and inhibitory potency of the ginsenosides, XueShuanTong was predicted to have high potential for OATP1B3-mediated drug interactions (attributed chiefly to ginsenoside $\mathrm{Rb}_{1}$ ) suggesting the need for further model-based determination of the interaction potential for XueShuanTong and, if necessary, a clinical drug interaction study. Increased awareness of ginsenosides' pharmacokinetics and XueShuanTong-drug interaction potential will help ensure the safe use of XueShuanTong and coadministered synthetic drugs.

Keywords: ginsenoside; cytochrome P450 3A; organic anion-transporting polypeptide 1B3; herbal medicine-drug interactions; XueShuanTong; Panax notoginseng

Acta Pharmacologica Sinica (2019) 40:1351-1363; https://doi.org/10.1038/s41401-019-0273-1

\section{INTRODUCTION}

Botanical products are ubiquitous worldwide, and there is a continued predisposition to the concurrent use of conventional synthetic drugs and botanical products. In China, many herbal medicines have been incorporated into the synthetic drugbased treatment of multifactorial diseases. In the USA and many European countries, the use of botanical dietary supplements or herbal medicinal products is prevalent, particularly in patient populations already exposed to conventional pharmacotherapy. Botanical products, either as herbal medicines or as dietary supplements, can lead to clinically important drug interactions, which often result from untoward alterations in absorption, disposition, and pharmacokinetics of the concomitant drugs. Pharmacokinetic interactions between grapefruit juice and felodipine and between St. John's wort and cyclosporine are textbook examples of clinically significant botanical product-drug interactions [1-6]. In addition, many compounds derived from botanical products have been shown to inhibit and induce drug metabolizing enzymes and transporters in vitro [7-12]. Pharmacokinetic botanical-drug interactions are widely recognized as an important issue and, when they occur, may lead to a reduced clinical response or increased toxicity. To this end, systematic research is essential for the identification and management of interaction-related clinical risk. However, the results from many prior investigations have been largely inconclusive or confusing, and their clinical relevance is insufficient to provide any useful guidance. Such investigations are often limited by poor understanding of the chemical basis responsible for and the mechanisms underlying botanical product-perpetrated interactions. This is because the chemical composition and human pharmacokinetics of test botanical products are limited or not characterized.

\footnotetext{
${ }^{1}$ State Key Laboratory of Drug Research, Shanghai Institute of Materia Medica, Chinese Academy of Sciences, Shanghai 201203 , China; ${ }^{2}$ University of Chinese Academy of Sciences, Beijing 100049, China and ${ }^{3}$ Second Affiliated Hospital, Tianjin University of Traditional Chinese Medicine, Tianjin 300250, China

Correspondence: Chuan Li (chli@simm.ac.cn) or Jun-ling Yang (yangjl@simm.ac.cn)
}

Received: 15 March 2019 Accepted: 11 June 2019

Published online: 29 July 2019 
In China, 'ginseng' refers to several different medicinal herbs of Panax species, including $P$. ginseng $C$. A. Meyer, $P$. notoginseng (Burk.) F. H. Chen, and P. quinquefolius L. The triterpene saponins ginsenosides, classified into 20(S)-protopanaxadiol-type (ppdtype), 20(S)-protopanaxatriol-type (ppt-type), and other types, are the major bioactive constituents of these ginseng herbs. Differences among Panax species in chemical composition, such as in the proportion and content levels of ginsenosides, may result in ginseng products being used for different conditions and may be associated with a differential drug interaction potential. Considerable speculation regarding ginseng-perpetrated drug interactions frequents the literature, but conclusive evidence for such interactions is lacking [13-19]. For instance, Malati et al. reported significantly reduced levels of systemic exposure to oral midazolam [a probe substrate for cytochrome P450 3A (CYP3A) phenotyping] after a 28-day oral administration of $P$. ginseng capsule ( $500 \mathrm{mg}$, b.i.d.; Vitamer, CA, USA) in healthy human subjects, suggesting possible induction of CYP3A by the botanical product [20]. However, another human study by Gurley et al. suggested that 28-day administration of the same $P$. ginseng capsule (500 mg, t.i.d.; Vitamer, CA, USA) did not significantly alter midazolam metabolism [21]. In addition to the approach of midazolam phenotyping being different between the two studies (i.e., Malati et al. using midazolam $\mathrm{AUC}_{0-\infty}$ to assess altered CYP3A activity vs. Gurley et al. using serum concentration ratio of $1^{\prime}$ hydroxymidazolam/midazolam at $1 \mathrm{~h}$ after dosing), information was not provided on interaction-related human pharmacokinetics of the test botanical products, as well as the quality consistency between the product lots used in the two studies. Investigation by $\mathrm{Hu}$ et al. indicates that metabolized, rather than unchanged, ginsenosides are the predominant circulating forms in humans orally receiving an extract of $P$. notoginseng roots and exhibit very large interindividual differences in plasma concentrations [22]. Recently, ginsenosides, particularly those of ppd-type and/or without a sugar attachment at C-20, were found to potently inhibit organic anion-transporting polypeptide (OATP)1B3 and, to a lesser extent, OATP1B1 [11, 12]. Further evaluation of potential OATP1Bmediated drug interactions with ginsenoside-containing herbal medicines requires human pharmacokinetic data of the ginsenosides present.

XueShuanTong, a sterile, nonpyrogenic, lyophilized solid for intravenous administration, is prepared from $P$. notoginseng roots (Chinese name, Sanqi) and approved by the Chinese National Medical Products Administration (NMPA; formerly the China Food and Drug Administration) as add-on therapy in the treatment of ischemic heart and cerebrovascular diseases. Several clinical studies have demonstrated the effectiveness of adding XueShuanTong to conventional treatment of the ischemic diseases [23-25]. XueShuanTong comprises ginsenosides, which are believed to be responsible for the medicine's therapeutic action. Given the common comedication of XueShuanTong with synthetic drugs and substantially higher systemic exposure to ginsenosides after intravenous administration than after oral administration (due to poor intestinal absorption of ginsenosides), drug interaction information is essential for therapies that include XueShuanTong. This investigation was designed to evaluate XueShuanTong-drug interaction potential, focusing on CYP3A induction and OATP1B inhibition, on the basis of understanding this herbal medicine's chemical composition and interactionrelated human pharmacokinetics.

\section{MATERIALS AND METHODS}

\section{Study design}

This investigation was designed to determine the potential for XueShuanTong-drug interactions. Based on literature-mined information and on findings from our earlier related studies, the investigation focused on XueShuanTong-mediated CYP3A induction and OATP1B3 inhibition. As the first step of investigation, the chemical composition and related lot-to-lot quality consistency of XueShuanTong were characterized by liquid chromatography/mass spectrometry. This was followed by a human pharmacokinetic study of ginsenosides from intravenously dosed XueShuanTong to identify herbal compounds considerably bioavailable for drug interactions and to characterize their pharmacokinetics/disposition that could influence the interaction potential. Two types of XueShuanTong-drug interaction study were performed: (1) evaluation of XueShuanTong's potential for CYP3A induction in human subjects with the results confirmed by a cell-based induction study using the bioavailable XueShuanTong compounds with respect to enzyme activity and mRNA level and (2) prediction of XueShuanTong's potential for OATP1B3 inhibition based on individual and joint inhibition of the transporter by the bioavailable XueShuanTong compounds and their time-related unbound levels of systemic exposure in humans. In the CYP3A induction study, possible influence of enzyme inhibition on the results was assessed in vivo and in vitro. In the OATP1B3 inhibition study, contributions of the ginsenosides to the interaction potential were compared.

\section{XueShuanTong, chemicals, and reagents}

Samples of five lots of XueShuanTong, with the Chinese NMPA drug ratification number of GuoYaoZhunZi-Z20025652, were obtained from Guangxi Wuzhou Pharmaceutical Group (Wuzhou, Guangxi Zhuang Autonomous Region, China). XueShuanTong is a sterile and nonpyrogenic lyophilized solid for intravenous administration. Each gram of XueShuanTong is prepared from $15.4 \mathrm{~g}$ of Sanqi ( $P$. notoginseng roots). XueShuanTong $(150 \mathrm{mg})$ is standardized to contain $14.3-22.5 \mathrm{mg}$ notoginsenoside $R_{1}, 54.0-84.0 \mathrm{mg}$ ginsenoside $\mathrm{Rg}_{1}, 7.5-12.0 \mathrm{mg}$ ginsenoside $\mathrm{Re}, 31.5-48.0 \mathrm{mg}$ ginsenoside $R b_{1}$, and not less than $0.7 \mathrm{mg}$ ginsenoside $\mathrm{Rd}$ and the sum of these ginsenosides is required to be within 128-167 mg. XueShuanTong from lot 13100513 was used in this study. A $5 \%$ glucose injection, with a Chinese NMPA drug ratification number of GuoYaoZhunZi-H12020021, was obtained from China Otsuka Pharmaceuticals (Dalian, Liaoning Province, China). Midazolam maleate tablet, with a Chinese NMPA drug ratification number of GuoYaoZhunzi-H20010311, was obtained from Roche Pharmaceuticals (Shanghai, China).

Ginsenosides $F_{2}, R_{1}, R b_{3}, R d, R e, R f, R g_{1}, R g_{3}$, compound- $K, 20$ (S)-protopanaxadiol, 20(S)-protopanaxatriol, and notoginsenoside $\mathrm{R}_{1}$ were obtained from the National Institutes for Food and Drug Control (Beijing, China); Ginsenoside $\mathrm{F}_{1}, \mathrm{Ra}_{1}, \mathrm{Ra}_{3}, \mathrm{Rg}_{2}, \mathrm{Rh}_{1}$, and notoginsenoside $F_{a}$ from Biopurify Phytochemicals (Chengdu, Sichuan Province, China); and 20-gluco-ginsenoside Rf from Fengshanjian (Kunming, Yunnan Province, China). The purity of these compounds exceeded $98 \%$. Midazolam, 1'-hydroxymidazolam, rifampin, $\beta$-nicotinamide adenine dinucleotide phosphate hydrate, glucose-6-phosphate monosodium salt, glucose-6phosphate dehydrogenase, and ketoconazole were obtained from Sigma-Aldrich (St. Louis, MO, USA). Estradiol-17 $\beta-D$-glucuronide $\left(E_{2} 17 \beta G\right)$ was obtained from Cayman Chemical (Ann Arbor, Ml, USA). HPLC grade methanol, formic acid, lithium acetate dehydrate, and dimethyl sulfoxide were also obtained from Sigma-Aldrich. Deionized water was purified using a Millipore Milli-Q Integral 3 (Milford, MA, USA).

Plateable cryopreserved primary human hepatocytes from three donors (lots IZT/Caucasian, HVN/Caucasian, and XSM/Hispanic) were obtained from BioIVT (Baltimore, MD, USA). Pooled human liver microsomes, high-viability cryohepatocyte recovery medium, cryohepatocyte plating medium, Matrigel matrix, and fetal bovine serum were purchased from Corning Gentest (Woburn, MA, USA). William's Medium E and cell maintenance supplements were obtained from Thermo Fisher Scientific (Grand Island, NY, USA). Rat tail collagen type I was obtained from Millipore (Temecula, CA, 
USA). An MTT-based in vitro toxicology assay kit was obtained from Sigma-Aldrich.

\section{Human studies}

Two human studies were performed at the National Clinical Research Center at the Second Affiliated Hospital of Tianjin University of Traditional Chinese Medicine (Tianjin, China). The study protocols were reviewed and approved by the ethics committee of clinical investigation at the hospital. The studies were registered at the Chinese Clinical Trials Registry (www.chictr. org) with a registration number of ChiCTR-ONRC-13003905 and carried out in accordance with the Declaration of Helsinki. Thirtysix healthy volunteers ( 24 men and 12 women) aged $21-33$ years (body mass index, $22.1 \pm 1.8 \mathrm{~kg} / \mathrm{m}^{2}$ ) were eligible to participate in this study. Evaluation of potential participants included a medical history, physical examination, and basic laboratory monitoring to rule out any medical conditions that could increase subject risk or affect study results. All participants were non-smokers and were not allowed to use any synthetic drugs (including oral contraceptives) or herbal medicines within 14 days prior to and during the period of study participation. They were not permitted to consume caffeine-containing products and alcoholic beverages $48 \mathrm{~h}$ prior to the study until the end of the study period. Additional exclusion criteria included a history of intolerance to any of the study medications. Informed consent was obtained from all participants.

In the first human study, an open-label study, 28 subjects were assigned into two groups (eight males and six females in each group) to characterize the pharmacokinetics of ginsenosides after intravenous dosing of XueShuanTong. For dosing, the XueShuanTong solid ( 250 or $500 \mathrm{mg}$ ) was dissolved in $250 \mathrm{~mL}$ of $5 \%$ glucose injection. A 2.5-h infusion of the resulting XueShuanTong solution was implemented using a ZNB-XB intelligent infusion pump (Beijing, China). Serial blood samples $(\sim 1 \mathrm{~mL}$ each time) were collected, from an antecubital vein catheter, before and $0.75,1.5$, 2.5 (just before terminating infusion), 2.67, 3, 3.5, 4.5, 6.5, 10.5, 24, 48 , and $72 \mathrm{~h}$ after starting the infusion of XueShuanTong at 250 or $500 \mathrm{mg} /$ person. Meanwhile, serial urine samples were also collected and weighed before and $0-3,3-6,6-10,10-24,24-48$, and $48-72 \mathrm{~h}$ after starting the infusion. Blood samples were heparinized and centrifuged at $3000 \times g$ for $10 \mathrm{~min}$ to obtain plasma fractions for liquid chromatography/mass spectrometrybased analysis. The freshly prepared plasma samples $(200 \mu \mathrm{L})$ were also dialyzed to determine unbound fractions of ginsenosides in plasma using a method by Guo et al. [26].

In the second human study, an open-label study with a fixed treatment order, eight other male subjects were recruited to determine the potential for induction of CYP3A by XueShuanTong and to characterize the pharmacokinetics of major circulating ginsenosides during repeated doses of XueShuanTong. On day 1, each subject received an oral dose of midazolam tablet at $7.5 \mathrm{mg}$. After a 72-h washout period, these subjects received repeated 2.5$\mathrm{h}$ infusions of XueShuanTong for 15 days (from day 4 to day 18) at $500 \mathrm{mg}$ per day. On days 4 and 18, each subject also received an oral dose of midazolam tablet at $7.5 \mathrm{mg}$ (just after terminating the infusion of XueShuanTong on that day). On day 1, serial blood samples ( 1 $\mathrm{mL}$ each time) were collected before and $0.17,0.5,1$, $2,4,8$, and $21.5 \mathrm{~h}$ after dosing midazolam; serial urine samples were also collected before and $0-3,3-6,6-10$, and $10-24 \mathrm{~h}$ after dosing. On days 4 and 18 , serial blood samples were collected before and $0.75,1.5,2.5$ (just before terminating the infusion of XueShuanTong), 2.67, 3, 3.5, 4.5, 6.5, 10.5, 24, 48 h (day 18 only), and $72 \mathrm{~h}$ (day 18 only) after starting the infusion; in addition, serial urine samples were collected and weighed before and $0-3,3-6$, $6-10,10-24,24-48 \mathrm{~h}$ (day 18 only), and $48-72 \mathrm{~h}$ (day 18 only) after starting the infusion. On days $6,8,10,12,14$, and 16 , blood samples were collected, before and $2.5 \mathrm{~h}$ (just before terminating the infusion of XueShuanTong) after starting the infusion of
XueShuanTong. Blood samples were heparinized and centrifuged at $3000 \times g$ for $10 \mathrm{~min}$ to obtain plasma fractions; no preservative was added into the urine samples. Plasma and urine samples were stored at $-70^{\circ} \mathrm{C}$ until analysis.

The liver function and kidney function of each subject in the second study were monitored on the day before the first dose of midazolam and also on days 11 and 23. Serum alanine aminotransferase, aspartate aminotransferase, total protein, albu$\mathrm{min} / \mathrm{globulin}$ ratio, total bilirubin, and direct bilirubin were monitored as liver function markers, while serum creatinine and blood urea nitrogen were monitored as kidney function markers.

In vitro assessment of CYP3A induction by XueShuanTong ginsenosides

Ginsenosides $R b_{1}(\mathbf{1})$, $R d(2)$, and $R g_{1}(31)$ and notoginsenoside $R_{1}$ (32) were assessed for their induction of CYP3A using cryopreserved human hepatocytes from three donors. Before use, the cells were rapidly thawed at $37^{\circ} \mathrm{C}$ and suspended in prewarmed CryoHepatocyte recovery medium. After centrifugation at $100 \times g$ for $10 \mathrm{~min}$, the supernatant was aspirated, and the cells were resuspended in CryoHepatocyte plating medium presupplemented with $10 \%$ fetal bovine serum and seeded at $6 \times 10^{4}$ cells/well in 96-well plates precoated with rat tail collagen type I. During the first $2 \mathrm{~h}$ of seeding, the cells in the plates were redistributed every $30 \mathrm{~min}$ by gently rocking the plates. After incubating for $4 \mathrm{~h}$, the medium was replaced with a maintenance medium (William's Medium E supplemented with $4 \%$ cell maintenance cocktail-B/ $100 \mathrm{nmol} / \mathrm{L}$ dexamethasone) and then maintained for $24 \mathrm{~h}$. The cells were then overlaid with Matrigel and incubated for $6 \mathrm{~h}$. This was followed by treating the cells, in triplicate, with the test ginsenosides [1/10/100 $\mu \mathrm{mol} / \mathrm{L}$, except for ginsenoside Rd (2) at $0.1 / 1 / 10 \mu \mathrm{mol} / \mathrm{L}$ and XueShuanTong at the marker concentrations $1 / 10 / 100 \mu \mathrm{mol} / \mathrm{L}$ of ginsenoside $\mathrm{Rg}_{1}$ (31) present], by freshly spiking each into the maintenance medium every $24 \mathrm{~h}$ over a period of $72 \mathrm{~h}$. After the treatment, the incubation medium was replaced with the maintenance medium prespiked with midazolam $(200 \mu \mathrm{mol} / \mathrm{L})$. After incubation for $30 \mathrm{~min}$, hydroxylation of midazolam was terminated by transferring the medium $(75 \mu \mathrm{L})$ into a microcentrifuge tube containing ice-cold acetonitrile (75 $\mu \mathrm{L})$. After centrifugation, the supernatant was analyzed by liquid chromatography/mass spectrometry to determine the concentration of the metabolite $1^{\prime}$-hydroxymidazolam. Using rifampin (20 $\mu \mathrm{mol} / \mathrm{L}$ ) in place of the test ginsenoside in the incubation served as the positive control, while using the same volume of solvent for dissolving ginsenoside in place of the ginsenoside served as the negative control. A ratio of enzyme activity, as indicated by the formation of 1 '-hydroxymidazolam, between the treated cells and the negative control was defined as a measure of the induction effect of the test ginsenoside. Such a ratio $\geq 2$ and the test compound's ratio $\geq 20 \%$ of the positive control's value indicated a positive result, from at least one of the three donors [27].

In addition, the ginsenosides (1, 2, 31, and 32) were also assessed with respect to cellular mRNA levels of CYP3A4 and CYP3A5, which were determined by SYBR-based quantitative realtime polymerase chain reaction (qRT-PCR) analysis. The relative quantity of the target mRNA was determined by the $\Delta \Delta \mathrm{Ct}$ method [28]. Using rifampin $(20 \mu \mathrm{mol} / \mathrm{L})$ in place of the test ginsenoside in the incubation served as the positive control, while using the same volume of solvent for dissolving ginsenoside in place of the ginsenoside served as the negative control. A ratio of the enzyme mRNA level between the treated cells and the negative control was defined as a measure of the induction effect of the test ginsenoside. Such a ratio $\geq 4$ and the test compound's ratio $\geq 20 \%$ of the positive control's value indicated a positive result, from at least one of the three donors [27].

Human hepatocytes were exposed to individual ginsenosides $\mathrm{Rb}_{1}(\mathbf{1} ; 1 / 10 / 100 \mu \mathrm{mol} / \mathrm{L}), \operatorname{Rd}(\mathbf{2} ; 0.1 / 1 / 10 \mu \mathrm{mol} / \mathrm{L})$, and $\mathrm{Rg}_{1}$ (31; $1 / 10 / 100 \mu \mathrm{mol} / \mathrm{L})$, notoginsenoside $R_{1}(\mathbf{3 2} ; 1 / 10 / 100 \mu \mathrm{mol} / \mathrm{L})$, 
and XueShuanTong [at the marker concentrations $1 / 10 / 100$ $\mu \mathrm{mol} / \mathrm{L}$ of ginsenoside $\mathrm{Rg}_{1}$ (31) present] at $37^{\circ} \mathrm{C}$ for $72 \mathrm{~h}$ to assess the effects of the test compounds and mixture on the cell mitochondria activity using an MTT-based in vitro toxicology assay kit according to the manufacturer's protocol.

In vitro assessment of CYP3A inhibition by XueShuanTong ginsenosides

Inhibition of CYP3A by ginsenosides $\mathrm{Rb}_{1}(\mathbf{1})$, $\mathrm{Rd}(\mathbf{2})$, and $\mathrm{Rg}_{1}(\mathbf{3 1})$, notoginsenoside $R_{1}(\mathbf{3 2})$, and XueShuanTong was assessed, in triplicate, by incubating a mixture $(100 \mu \mathrm{L})$ that contained pooled human liver microsomes $(0.1 \mathrm{mg}$ protein $/ \mathrm{mL})$, midazolam $(2.5$ $\mu \mathrm{mol} / \mathrm{L})$, the test ginsenoside, an NADPH-generating system, and potassium phosphate buffer $(100 \mathrm{mmol} / \mathrm{L}, \mathrm{pH} 7.4)$ at $37^{\circ} \mathrm{C}$ for 5 min. After incubation, the samples were analyzed, by liquid chromatography/mass spectrometry, to determine the formation of the metabolite $1^{\prime}$-hydroxymidazolam. The final concentration of the ginsenoside was $100 \mu \mathrm{mol} / \mathrm{L}$, while that of XueShuanTong was the marker concentration $100 \mu \mathrm{mol} / \mathrm{L}$ of ginsenoside $\mathrm{Rg}_{1}$ present. Ketoconazole $(5 \mu \mathrm{mol} / \mathrm{L})$, in place of the test ginsenoside in the incubation, was used as the positive control, while the same volume of solvent for dissolving ginsenoside in place of the ginsenoside was used as the negative control.

In vitro assessment of joint inhibition of OATP1B by XueShuanTong ginsenosides

Joint inhibition of OATP1B by ginsenosides $\mathrm{Rb}_{1}(\mathbf{1}), \mathrm{Rd}(\mathbf{2})$, and $\mathrm{Rg}_{1}$ (31) and notoginsenoside $R_{1}$ (32) was assessed in vitro, and the data were processed using the Chou-Talalay method [29]. Ginsenosides $\mathrm{Rb}_{1}(\mathbf{1}), \mathrm{Rd}(\mathbf{2})$, and $\mathrm{Rg}_{1}$ (31) and notoginsenoside $R_{1}$ (32) were mixed at a ratio of 10:1:100:10, which is similar to that of the unbound plasma $C_{\max }$ on day 18 of repeatedly dosing intravenous XueShuanTong at the label dose $500 \mathrm{mg} / \mathrm{day}$. This ratio was held constant, while the total ginsenoside concentrations were varied by serial dilution. Inhibition of the transporter by the individual ginsenosides and joint inhibition by the ginsenoside combinations were assessed using transiently transfected HEK-293 cells expressing the transporter, with $E_{2} 17 \beta G$ as the substrate [11]. The joint inhibition was characterized with respect to the combination index $(\mathrm{Cl})$, which was calculated using Combosyn Compusyn software (version 1.0; Paramus, NJ, USA). A Cl value of, $<0.9,0.9-1.1$, or $>1.1$ indicated that the joint inhibition was synergistic, additive, or antagonistic, respectively. The $\mathrm{IC}_{50}$ values of individual ginsenosides were estimated using the method reported by Jiang et al. [11].

\section{Analysis of XueShuanTong samples for ginsenosides}

A Waters Synapt G2 high definition time-of-flight mass spectrometer (Manchester, UK), interfaced via a LockSpray source with a Waters Acquity ultraperformance liquid chromatographic separation module (Milford, MA, USA), was used for detection, characterization, and grading of ginsenosides present in samples of XueShuanTong. Sample preparation was performed by dissolving XueShuanTong in water. Chromatographic separation was achieved on a $1.7 \mu \mathrm{m}$ Waters BEH C18 column $(100 \mathrm{~mm} \times 2.1 \mathrm{~mm}$ i.d.; $45^{\circ} \mathrm{C}$; Dublin, Ireland) with a mobile phase $(0.3 \mathrm{~mL} / \mathrm{min})$, consisting of solvents $A$ (methanol/water, 1:99, v/v; containing 1 $\mathrm{mmol} / \mathrm{L}$ formic acid) and $B$ (methanol/water, 99:1, v/v; containing $1 \mathrm{mmol} / \mathrm{L}$ formic acid). A 42-min gradient program was used, consisting of $0-2$ min at $2 \%$ B, 2-32 min from $2 \%$ to $98 \%$ B, 32-37 min at $98 \% \mathrm{~B}$, and $37-42 \mathrm{~min}$ at $2 \% \mathrm{~B}$. The mass spectrometer was operated in resolution mode and externally calibrated with sodium formate $(5 \mathrm{mmol} / \mathrm{L} ; 10 \mu \mathrm{L} / \mathrm{min})$ over a range of $\mathrm{m} / \mathrm{z}$ 50-1500; mass shifts during acquisition were corrected with leucine encephalin ( $\mathrm{m} / \mathrm{z}$ 554.2615; the negative ion mode). $\mathrm{MS}^{\mathrm{E}}$ data acquisition (in centroid mode, $\mathrm{m} / \mathrm{z}$ 50-1500) was achieved simultaneously using a trap collision energy of $3 \mathrm{~V}$ and a trap collision energy ramp of $30-50 \mathrm{~V}$ with a scan time of $0.4 \mathrm{~s}$.
Literature mining was performed to generate a preanalysis Sanqi ginsenoside list, which contained information, such as the compounds' names, structures, accurate molecular masses, electrospray ionization profiles, collision-induced fragmentation profiles, and literature sources. Ginsenosides present in XueShuanTong were initially detected based on accurate molecular masses and electrospray ionization profiles. The suspected ginsenosides were characterized by comparing their accurate molecular masses, fragmentation profiles, and chromatographic retention times with those of the reference standards. When such standards were not available, the characterization was based on comparison with the literature-mined information, such as the mass spectrometric data for the suspected ginsenosides and the chromatographic elution order with other related compounds. Follow-up quantification of the characterized ginsenosides in XueShuanTong samples was based on calibration with their respective reference standards or, when their respective reference standards were not available, with a structurally similar reference standard.

\section{Analysis of human samples for unchanged and metabolized} ginsenosides

To analyze unchanged and metabolized ginsenosides in human subjects receiving XueShuanTong, plasma and urine samples were prepared with methanol at a volumetric sample-to-methanol ratio of $1: 3$ and then centrifuged. After the supernatants were reduced to dryness under reduced pressure, the residues were reconstituted with $50 \%$ methanol and then analyzed by liquid chromatography/mass spectrometry. The chromatographic and mass spectrometric conditions were the same as those described for analysis of XueShuanTong samples for ginsenosides. Detection and characterization of unchanged ginsenosides in human plasma and urine samples after dosing XueShuanTong were achieved using the characterized XueShuanTong sample (with respect to ginsenosides) as reference standard. Detection and characterization of metabolites of ginsenosides in these human plasma and urine samples were achieved using our earlier characterized human plasma and urine samples (with respect to unchanged and metabolized ginsenosides), obtained after oral dosing of a Sanqi extract, as described by Hu et al. [22]. Follow-up quantification of the characterized ginsenosides and their metabolites in human samples was based on calibration with their respective reference standards or, when their respective reference standards were not available, with a structurally similar reference standard. Due to assay sensitivity, some minor ginsenosides and the metabolites were detected and characterized using an AB Sciex API 4000 Q Trap mass spectrometer (Toronto, Canada), interfaced via a Turbo V ion source with an Agilent 1290 Infinity II liquid chromatograph (Waldbronn, Germany), and the mobile phase contained 0.025 $\mathrm{mmol} / \mathrm{L}$ lithium acetate.

Quantification of ginsenosides, midazolam, 1'-hydroxymidazolam, and $E_{2} 17 \beta G$ in study samples

Concentrations of the major circulating ginsenosides, i.e., ginsenosides $\mathrm{Rb}_{1}(\mathbf{1})$, $\mathrm{Rd}(\mathbf{2})$, and $\mathrm{Rg}_{1}$ (31) and notoginsenoside $\mathrm{R}_{1}(\mathbf{3 2})$, in human plasma and urine samples after dosing XueShuanTong were determined using the $A B$ Sciex mass spectrometer interfaced with the Agilent liquid chromatograph. Methods for sample preparation were the same as those used in the detection of unchanged and metabolized ginsenosides in human samples. Chromatographic separation was achieved on a $5 \mu \mathrm{m}$ Agilent ZORBAX Eclipse Plus $\mathrm{C}_{18}$ column $(50 \mathrm{~mm} \times 2.1 \mathrm{~mm}$ i.d.; Santa Clara, CA, USA) with a mobile phase $(0.35 \mathrm{~mL} / \mathrm{min})$, consisting of solvents A (methanol/water, 1:99, v/v; containing $0.025 \mathrm{mmol} / \mathrm{L}$ lithium acetate and $1 \mathrm{mmol} / \mathrm{L}$ formic acid) and B (methanol/water, 99:1, v/ $\mathrm{v}$; containing $0.025 \mathrm{mmol} / \mathrm{L}$ lithium acetate and $1 \mathrm{mmol} / \mathrm{L}$ formic acid). An 8-min gradient program was used, consisting of $0-2$ min from $20 \%$ B to $70 \%$ B, 2-5 min from $70 \%$ B to $90 \%$ B, 5-7 min at 
$90 \% \mathrm{~B}, 7-7.1 \mathrm{~min}$ from $90 \% \mathrm{~B}$ to $20 \% \mathrm{~B}$, and $7.1-8 \mathrm{~min}$ at $20 \% \mathrm{~B}$. The mass spectrometer was operated in the positive ion mode to generate lithiated molecules. Optimal precursor-to-product ion pairs for multiple reaction monitoring of ginsenosides $R b_{1}(\mathbf{1}), \mathrm{Rd}$ (2), and $\mathrm{Rg}_{1}$ (31) and notoginsenoside $\mathrm{R}_{1}$ (32) were $\mathrm{m} / \mathrm{z} 1115 \rightarrow 349$ (collision energy, $81 \mathrm{~V}), 953 \rightarrow 773(63 \mathrm{~V})$, and $807 \rightarrow 627(51 \mathrm{~V})$ and $939 \rightarrow 319(73 \mathrm{~V})$, respectively. Matrix-matched calibration curves were constructed with the reference standards using weighted (1/ $X$ or $\left.1 / X^{2}\right)$ linear regression of the peak areas $(Y)$ of the analytes against the corresponding nominal analytes' concentrations ( $X$ : $1.37,4.12,12.4,37.0,111,333,1000,3000,5000$, and $10,000 \mathrm{nmol} /$ L). The calibration curves showed good linearity $(R>0.99)$. Although no internal standard was used, assay validation, implemented according to the European Medicines Agency Guideline on Bioanalytical Method Validation (2012; www.ema. europa.eu), demonstrated that the assays were reliable and reproducible for intended use. The lower limits of quantification were $37.0,4.12,4.12$, and $4.12 \mathrm{nmol} / \mathrm{L}$ for ginsenosides $\mathrm{Rb}_{1}(\mathbf{1}), \mathrm{Rd}$ (2), and $R_{1}(\mathbf{3 1})$ and notoginsenoside $R_{1}(32)$, respectively, and the upper limits of quantification were, $10000,5000,5000$, and $3000 \mathrm{nmol} / \mathrm{L}$, respectively. The intraday accuracy and precision were $89 \%-115 \%$ and $2 \%-18 \%$, respectively, while the interday values were $86-113 \%$ and $2 \%-12 \%$, respectively. The coefficients of variation of matrix factors were $1.2 \%-14.4 \%$. The recovery of sample preparation was concentration-independent and was 97\%-101\%, 76\%-86\%, 80\%-93\%, and 93\%-99\% for ginsenosides $\mathrm{Rb}_{1}$ (1), $\mathrm{Rd}$ (2), and $\mathrm{Rg}_{1}$ (31) and notoginsenoside $\mathrm{R}_{1}$ (32), respectively. The carryover in the blank sample following the upper limits of quantification was negligible for these ginsenosides.

Concentrations of midazolam and 1'-hydroxymidazolam in human plasma samples and in incubation samples (obtained from in vitro studies) were determined using an assay similar to that for measurement of the ginsenosides in human plasma samples, except for some chromatographic and mass spectrometric conditions. In brief, the mobile phase for chromatographic separation consisted of solvents A (methanol/water, 1:99, v/vi containing $1 \mathrm{mmol} / \mathrm{L}$ formic acid) and $\mathrm{B}$ (methanol/water, 99:1, v/v; containing $1 \mathrm{mmol} / \mathrm{L}$ formic acid) and was delivered at $0.3 \mathrm{~mL} /$ min. A 6.5-min gradient program was used, consisting of $0-0.5$ $\mathrm{min}$ at $5 \% \mathrm{~B}, 0.5-2.5 \mathrm{~min}$ from $5 \% \mathrm{~B}$ to $90 \% \mathrm{~B}, 2.5-3 \mathrm{~min}$ from $90 \%$ $B$ to $95 \%$ B, 3-5 min at $95 \%$ B, 5-5.1 min from $95 \%$ B to $5 \%$, and $5.1-6.5 \mathrm{~min}$ at $5 \% \mathrm{~B}$. The mass spectrometer was operated in the positive ion mode to generate protonated molecules. Optimal precursor-to-product ion pairs for multiple reaction monitoring of midazolam and 1'-hydroxymidazolam were $\mathrm{m} / \mathrm{z} 326 \rightarrow 291$ (collision energy, $30 \mathrm{~V})$ and $342 \rightarrow 324(30 \mathrm{~V})$, respectively. Matrixmatched calibration curves were constructed with the reference standards midazolam and 1'-hydroxymidazolam using weighted $(1 / X)$ linear regression of the peak areas $(Y)$ of the analytes against the corresponding nominal analytes' concentrations ( $X: 1.37,4.12$, $12.4,37,111,333,500$, and $1000 \mathrm{nmol} / \mathrm{L})$. The lower limits of quantification were 1.37 and $4.12 \mathrm{nmol} / \mathrm{L}$ for midazolam and $1^{\prime}$ hydroxymidazolam, respectively, and the upper limits of quantification were $1000 \mathrm{nmol} / \mathrm{L}$ for both. No internal standard was used; the intraday accuracy and precision were $94.7 \%-103.6 \%$ and $3.4 \%-10.7 \%$, respectively, while the interday values were $96.6 \%-101.0 \%$ and $2.9 \%-5.1 \%$, respectively. The recovery of sample preparation was $75 \%-98 \%$ and $83 \%-97 \%$ for midazolam and 1'-hydroxymidazolam, respectively; the carryover in the blank sample following the upper limits of quantification was negligible.

The assay for quantification of $E_{2} 17 \beta G$ in OATP1B-inhibition study samples was the same as that described by Jiang et al. [11].

\section{Data processing}

To understand the chemical composition of XueShuanTong and highlight the important ginsenosides present, the ginsenosides detected and characterized were first ranked according to their respective daily doses, which were calculated as the product of the ginsenoside content level in XueShuanTong and the injection's label daily dose $500 \mathrm{mg} /$ day. These ginsenosides were then graded into different levels according to their daily doses, i.e., $>10,1-10$, and $<1 \mu \mathrm{mol} /$ day.

Pharmacokinetic parameters of ginsenosides $\mathrm{Rb}_{1}(\mathbf{1}), \mathrm{Rd}(\mathbf{2})$, and $\mathrm{Rg}_{1}$ (31) and notoginsenoside $\mathrm{R}_{1}$ (32) were estimated by noncompartmental analysis using Innaphase Kinetica software package (version 5.0; Philadelphia, PA, USA). The potential for XueShuanTong ginsenosides to perpetrate drug interactions on OATP1B1/3 was estimated using equation 1 :

$\mathrm{DDI}$ index $=\left(C_{\max } \times f_{\mathrm{u}-\text { plasma }}\right) / \mathrm{IC}_{50}$

where $C_{\max }$ is the maximum total (unbound plus bound) plasma concentration, $f_{\text {u-plasma }}$ is the unbound fraction in plasma, and $\mathrm{IC}_{50}$ is the in vitro half maximum inhibitory concentration.

Pharmacokinetic parameters of midazolam and 1'-hydroxymidazolam were evaluated for statistically significant differences between values for days 1 and 4, between those for days 1 and 18, and between those for days 4 and 18 by the paired, two-tailed $t$ test. Statistical analysis was performed using IBM SPSS Statistics software (version 19.0; IBM, Chicago, IL, USA). $P<0.05$ was considered the minimum level of statistical significance. All data are expressed as the mean \pm standard deviation.

\section{RESULTS}

Ginsenosides detected in XueShuanTong and related lot-to-lot quality consistency

A total of 50 ginsenosides were detected and characterized in XueShuanTong: 14 ppd-type ginsenosides (1-14), 18 ppt-type ginsenosides (31-48), and 18 ginsenosides of other types (51-68) (Fig. 1 and Supplementary Table S1). The ginsenosides were graded, with respect to their compound doses, into three levels: $>10 \mu \mathrm{mol} /$ day [ginsenoside $\mathrm{Rb}_{1}$ (1), ginsenoside $\mathrm{Rg}_{1}$ (31), notoginsenoside $R_{1}(32)$, and ginsenoside $\left.\operatorname{Re}(33)\right], 1-10 \mu \mathrm{mol} /$ day [ginsenoside $\mathrm{Rd}(\mathbf{2})$, notoginsenoside $\mathrm{Fa}(\mathbf{3})$, ginsenoside $\mathrm{Ra}_{3}$ (4), notoginsenoside $R_{4}$ (5), ginsenoside $R_{1}$ (34), 20-glucoginsenoside $\mathrm{Rf}$ (35), ginsenoside $\mathrm{Rg}_{2}$ (36) and notoginsenoside $\mathrm{M} / \mathrm{N} / \mathrm{R}_{3} / \mathrm{R}_{6}$ isomer-3 (37)], and $<1 \mu \mathrm{mol} /$ day [the remaining 38 minor ginsenosides (6-14, 38-48, and 51-68)]. Neither 20(S)protopanaxadiol (the aglycone of ppd-type ginsenosides) nor 20 (S)-protopanaxatriol (the aglycone of ppt-type ginsenosides) was detected in XueShuanTong. The sum of doses of the four ginsenosides (1 and 31-33; compound dose, $>10 \mu \mathrm{mol} /$ day) accounted for $92.3 \%$ of the total dose of XueShuanTong ginsenosides, whereas such sum for eight ginsenosides (2-5 and 34-37; $1-10 \mu \mathrm{mol} / \mathrm{day}$ ) and for the remaining 38 minor ginsenosides (6-14, 38-48, and 51-68; <1 $\mathrm{mmol} / \mathrm{day})$ accounted for $5.0 \%$ and $2.7 \%$ of the total dose of XueShuanTong ginsenosides, respectively. XueShuanTong exhibited lot-to-lot variability of $4.4 \%-10.2 \%$ for the ginsenosides (1 and 31-33) and $7.3 \%-36.2 \%$ for the ginsenosides (2-5 and 34-37).

Human systemic exposure and pharmacokinetics of ginsenosides after dosing XueShuanTong

All 36 human subjects completed their scheduled treatment. As expected, subjects in the second human study experienced sedation following midazolam administration. No subject reported an unanticipated or serious adverse effect associated with XueShuanTong administration.

As shown in Fig. 2 and Supplementary Table S2, a total of 11 circulating ginsenosides, i.e., five ppd-type (1-5) and six ppt-type ginsenosides (31-36), were detected and characterized in human subjects after intravenous dosing of XueShuanTong. Circulating ginsenosides generally occurred in a compound dose-dependent manner; the minor ginsenosides (compound dose, $<1 \mu \mathrm{mol} /$ day) 

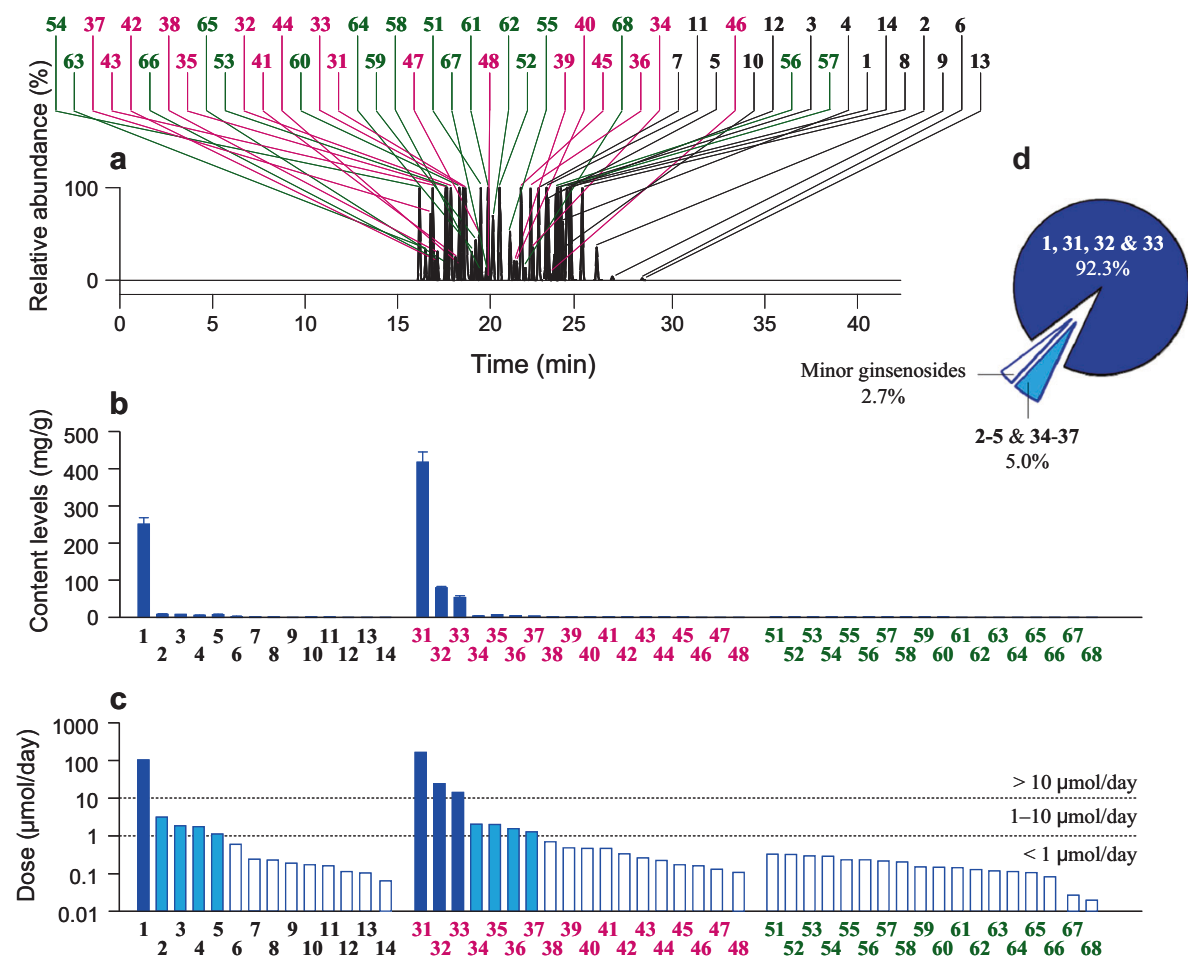

Compound ID

Fig. 1 Ginsenosides, detected by mass spectrometry, in XueShuanTong. (a) Stacked chromatograms of ginsenosides in a typical sample of XueShuanTong. (b) Mean content levels of ginsenosides in five lot-samples of XueShuanTong. (c) Doses of ginsenosides from the XueShuanTong lot used in the human pharmacokinetic study at the label dose $500 \mathrm{mg} /$ day. (d) Percentage daily doses of ginsenosides ( $>10$ $\mu \mathrm{mol} /$ day, comprising 1 and 31-33; 1-10 $\mu \mathrm{mol} /$ day, comprising 2-5 and 34-37; and $<1 \mu \mathrm{mol} /$ day, comprising 6-14, 38-48, and 51-68) in the total daily dose of all the ginsenosides detected in XueShuanTong. The names of XueShuanTong ginsenosides as indicated by compound IDs are shown in Supplementary Table S1
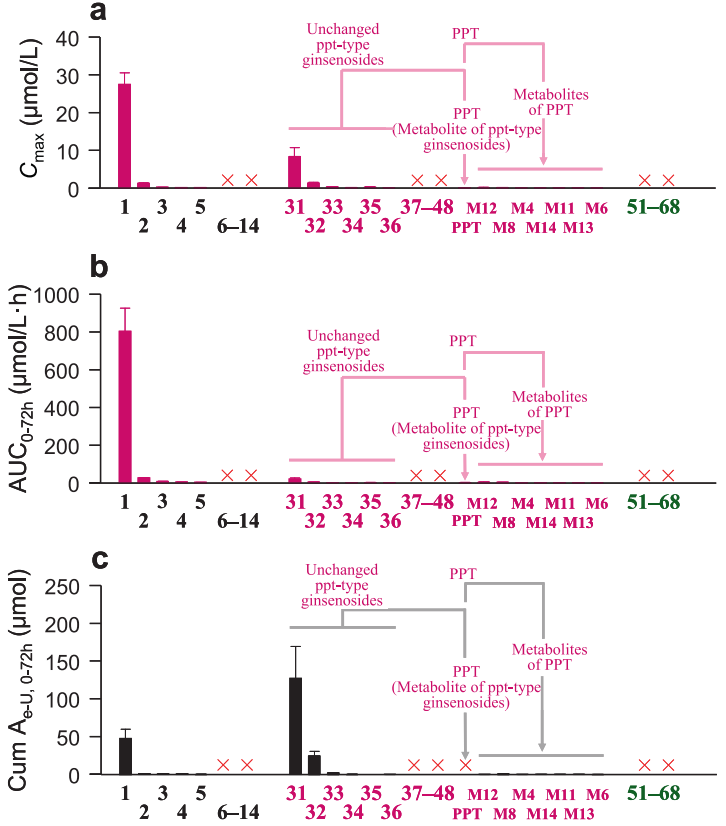

Compound/Metabolite ID

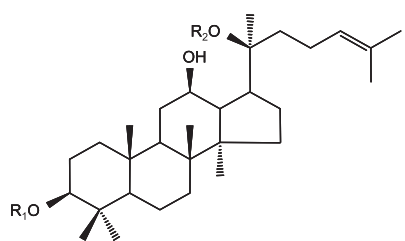

$\mathrm{R}_{1} \quad \mathrm{R}_{2}$

Ginsenoside $\mathrm{Rb}_{1}(\mathbf{1}) \quad-$ Glc-Glc $\quad-$ Glc-Glc Ginsenoside Rd(2) -Glc-Glc -Glc

Fig. 2 Systemic exposure (a, b) to and renal excretion (c) of ginsenosides, unchanged and metabolized, in human subjects ( $\mathrm{m} 9-\mathrm{m} 16$ and $\mathrm{f7}-$ f12) who received an intravenous infusion of XueShuanTong at $500 \mathrm{mg} / \mathrm{day}$, along with chemical structures of the major circulating ginsenosides. The unchanged ginsenosides are ranked in the same order as in Fig. $1 \mathrm{~b}, \mathrm{c}$, along with their detected metabolites. The symbol " $X$ " (in red) denotes a ginsenoside or metabolite that was not detected. The names of XueShuanTong ginsenosides, unchanged and metabolized, as indicated by compound/metabolite IDs, are shown in Supplementary Table S2. Glc glucopyranosyl; Xyl xylopyranosyl 
Table 1. Pharmacokinetics of ginsenosides $R b_{1}(\mathbf{1}), R d(\mathbf{2})$, and $R g_{1}(\mathbf{3 1})$ and notoginsenoside $R_{1}(\mathbf{3 2})$ in human subjects who received intravenous XueShuanTong

\begin{tabular}{|c|c|c|c|c|c|c|c|c|}
\hline Compound (ID) & $C_{\max }(\mu \mathrm{mol} / \mathrm{L})$ & $A \cup C_{0-t}(\mu \mathrm{mol} / L \cdot h)$ & $A \cup C_{0-\infty}(\mu \mathrm{mol} / \mathrm{L} \cdot h)$ & $t_{1 / 2}(\mathrm{~h})$ & $\begin{array}{l}\mathrm{CL}_{\text {tot, } p} \\
(\mathrm{~mL} / \mathrm{h} / \mathrm{kg})\end{array}$ & $V_{\mathrm{ss}}(\mathrm{mL} / \mathrm{kg})$ & $f_{\mathrm{u}}(\%)$ & $f_{\mathrm{e}-\mathrm{u}}(\%)$ \\
\hline \multicolumn{9}{|c|}{ Human study 1 (dose, $250 \mathrm{mg}$ XueShuanTong per person): subject ID, m1-m8 (male); body weight, 53.0-88.8 kg; age, 21-32 years old) } \\
\hline Ginsenoside Rd (2) & $0.7 \pm 0.2$ & $13.8 \pm 2.3$ & $49.7 \pm 25.0$ & $175.9 \pm 83.0$ & $1.8 \pm 0.6$ & $142.4 \pm 25.0$ & $2.1 \pm 0.8$ & $20.1 \pm 2.6$ \\
\hline Ginsenoside $\mathrm{Rg}_{1}$ (31) & $3.6 \pm 0.5$ & $7.8 \pm 0.9$ & $7.9 \pm 0.9$ & $1.3 \pm 0.2$ & $278.9 \pm 41.6$ & $338.8 \pm 35.5$ & $98.3 \pm 3.7$ & $42.1 \pm 4.5$ \\
\hline Notoginsenoside $\mathrm{R}_{1}$ (32) & $0.7 \pm 0.1$ & $1.6 \pm 0.2$ & $1.6 \pm 0.2$ & $1.4 \pm 0.2$ & $204.5 \pm 28.8$ & $283.2 \pm 30.2$ & $90.0 \pm 10.6$ & $49.4 \pm 6.8$ \\
\hline Ginsenoside $\mathrm{Rb}_{1}(\mathbf{1})$ & $19.5 \pm 2.2$ & $492.4 \pm 46.7$ & $691.1 \pm 65.9$ & $39.9 \pm 4.7$ & $1.5 \pm 0.1$ & $82.4 \pm 13.3$ & $1.1 \pm 0.3$ & $42.7 \pm 13.2$ \\
\hline Ginsenoside Rd (2) & $0.8 \pm 0.1$ & $14.2 \pm 2.5$ & $36.1 \pm 15.6$ & $133.9 \pm 112.4$ & $2.5 \pm 0.5$ & $143.0 \pm 24.0$ & $1.0 \pm 0.5$ & $18.6 \pm 4.2$ \\
\hline Ginsenoside $\mathrm{Rg}_{1}$ (31) & $5.0 \pm 0.6$ & $12.8 \pm 3.1$ & $12.8 \pm 3.2$ & $1.3 \pm 0.3$ & $219.4 \pm 64.4$ & $272.3 \pm 43.0$ & $83.5 \pm 8.2$ & $45.1 \pm 13.4$ \\
\hline Notoginsenoside $\mathrm{R}_{1}$ (32) & $1.0 \pm 0.1$ & $2.4 \pm 0.6$ & $2.5 \pm 0.6$ & $1.3 \pm 0.3$ & $174.5 \pm 47.3$ & $253.0 \pm 37.4$ & $78.5 \pm 17.8$ & $48.5 \pm 13.2$ \\
\hline \multicolumn{9}{|c|}{ Human study 1 (dose, $500 \mathrm{mg}$ XueShuanTong per person): subject ID, m9-m16 (male); body weight, 57.0-78.0 kg; age, 25-32 years old) } \\
\hline \multicolumn{9}{|c|}{ Human study 1 (dose, $500 \mathrm{mg}$ XueShuanTong per person): subject ID, f7-f12 (female); body weight, 48.7-66.8 kg; age, 21-27 years old) } \\
\hline Ginsenoside $\mathrm{Rb}_{1}(\mathbf{1})$ & $28.9 \pm 3.2$ & $887.4 \pm 97.5$ & $1269.9 \pm 206.6$ & $41.0 \pm 7.6$ & $1.5 \pm 0.3$ & $87.5 \pm 9.7$ & $1.9 \pm 0.2$ & $44.5 \pm 17.5$ \\
\hline Ginsenoside Rd (2) & $1.2 \pm 0.1$ & $26.4 \pm 2.2$ & $68.6 \pm 29.9$ & $119.7 \pm 82.0$ & $2.2 \pm 0.3$ & $161.2 \pm 19.2$ & $1.5 \pm 0.7$ & $17.5 \pm 6.5$ \\
\hline Ginsenoside $\mathrm{Rg}_{1}$ (31) & $7.7 \pm 0.8$ & $20.7 \pm 2.7$ & $20.8 \pm 2.8$ & $1.3 \pm 0.1$ & $240.1 \pm 33.5$ & $354.9 \pm 26.8$ & $97.5 \pm 3.0$ & $53.8 \pm 17.5$ \\
\hline Notoginsenoside $\mathrm{R}_{1}$ (32) & $1.5 \pm 0.2$ & $4.1 \pm 0.5$ & $4.1 \pm 0.5$ & $1.5 \pm 0.1$ & $184.8 \pm 24.9$ & $311.6 \pm 25.8$ & $94.5 \pm 6.7$ & $62.0 \pm 18.1$ \\
\hline \multicolumn{9}{|c|}{ Human study 2 (dose, $500 \mathrm{mg}$ XueShuanTong once daily; day 4): subject ID, m17-m24 (male); body weight, 57.4-73.8 kg; age, 25-33 years old) } \\
\hline Ginsenoside $\mathrm{Rb}_{1}(\mathbf{1})$ & $23.0 \pm 5.8$ & $245.1 \pm 25.1$ & $745.0 \pm 236.6$ & - & $2.1 \pm 0.8$ & $143.3 \pm 20.3$ & - & - \\
\hline Ginsenoside Rd (2) & $1.3 \pm 0.3$ & $11.0 \pm 1.4$ & $20.5 \pm 5.8$ & - & $4.5 \pm 1.5$ & $122.3 \pm 15.2$ & - & - \\
\hline Ginsenoside $\mathrm{Rg}_{1}$ (31) & $6.9 \pm 1.1$ & $16.9 \pm 1.6$ & $17.0 \pm 1.6$ & $1.4 \pm 0.2$ & $268.5 \pm 33.0$ & $342.0 \pm 53.8$ & - & - \\
\hline Notoginsenoside $\mathrm{R}_{1}$ (32) & $1.3 \pm 0.2$ & $3.4 \pm 0.3$ & $3.4 \pm 0.3$ & $1.4 \pm 0.1$ & $205.0 \pm 28.9$ & $296.0 \pm 42.5$ & - & - \\
\hline
\end{tabular}

The details of human pharmacokinetic study are described in "Materials and methods" section (Human studies). The data represent mean \pm standard deviation $C_{\max }$ maximum plasma concentration, $A \cup C_{0-t}$ area under the plasma concentration-time curve from 0 to last measured time point, $A \cup C_{0-\infty}$, area under the plasma concentration-time curve from 0 to infinity, $t_{1 / 2}$, terminal half-life, $\mathrm{CL}_{\text {tot, }}$ total plasma clearance, $V_{\mathrm{ss}}$ apparent volume of distribution at steady state, $f_{\mathrm{u}}$ unbound fraction in plasma, $f_{\mathrm{e}-\mathrm{u}}$ fraction of dose excreted into urine

were negligibly or not detected in plasma samples. Ppd-type ginsenosides $R b_{1}(\mathbf{1})$ and $R d(2)$ and ppt-type ginsenoside $R g_{1}$ (31) and notoginsenoside $R_{1}$ (32) were the major circulating XueShuanTong compounds. In addition to the unchanged ginsenosides, eight circulating metabolites of ppt-type ginsenosides were also detected; they were the deglycosylated metabolite 20(S)protopanaxatriol (PPT) and its further oxidized metabolites, $\mathbf{M}_{\mathbf{4}}$ $\mathbf{M}_{\mathbf{6}}, \mathbf{M}_{\mathbf{8}}$, and $\mathbf{M}_{\mathbf{1 1}}-\mathbf{M}_{\mathbf{1 4}}$. These metabolites were minor circulating XueShuanTong compounds compared with unchanged ppt-type ginsenoside $\mathrm{Rg}_{1}$ (31) and notoginsenoside $\mathrm{R}_{1}$ (32) (Fig. 2). All the unchanged circulating ginsenosides were also detected in human urine samples. In addition to the circulating metabolites (PPT, $\mathbf{M}_{\mathbf{4}}$, $\mathbf{M}_{\mathbf{6}}, \mathbf{M}_{\mathbf{8}}$, and $\left.\mathbf{M}_{\mathbf{1 1}}-\mathbf{M}_{\mathbf{1 4}}\right)$, more metabolites of PPT $\left(\mathbf{M}_{\mathbf{3}}, \mathbf{M}_{\mathbf{5}}\right.$, and $\left.\mathbf{M}_{\mathbf{7}}\right)$ were also detected in urine samples, but all at very low levels compared with those of ginsenoside $\mathrm{Rg}_{1}$ (31) and notoginsenoside $R_{1}$ (32). Such metabolites of ppd-type ginsenosides were not detected in plasma and urine samples after dosing XueShuanTong. Figure 2 shows the chemical structures of ginsenosides (1, 2, 31, and 32) that are considerably bioavailable for XueShuanTong-drug interactions.
Table 1 summarizes the human pharmacokinetics of ginsenosides $R b_{1}(\mathbf{1}), R d(2)$, and $R g_{1}(\mathbf{3 1})$ and notoginsenoside $R_{1}(\mathbf{3 2})$ after dosing XueShuanTong; Fig. 3 shows their total (unbound plus bound) and unbound plasma concentrations over time after a single dose of XueShuanTong at 250 or $500 \mathrm{mg} /$ person (human study 1). The total plasma concentrations of these ginsenosides over time after dosing XueShuanTong at $500 \mathrm{mg} /$ day on days 4 and 18 (human study 2) are shown in Supplementary Fig. S1. These ginsenosides exhibited increased maximum plasma total concentration $\left(C_{\max }\right)$ and area under the plasma concentration-time curve from 0 to infinity $\left(A U C_{0-\infty}\right)$ as the XueShuanTong dose increased from 250 to $500 \mathrm{mg} /$ person. After correcting the compound doses for the subject's body weight, there was no significant gender difference in $C_{\max }$ or $A U C_{0-\infty}$ of the ginsenosides $(P=0.07-0.95)$, except for ginsenoside $\mathrm{Rd}$ (2) and notoginsenoside $R_{1}$ (32), which exhibited significant, but slight, gender differences in $C_{\max }$ (both at $500 \mathrm{mg}$ XueShuanTong per person; $P<0.05)$. There were notable intercompound differences in terminal half-lives $\left(t_{1 / 2}\right)$ between the ppd-type ginsenosides (1 and $\mathbf{2}$ ) and the ppt-type ginsenosides (31 and 32); the differences 

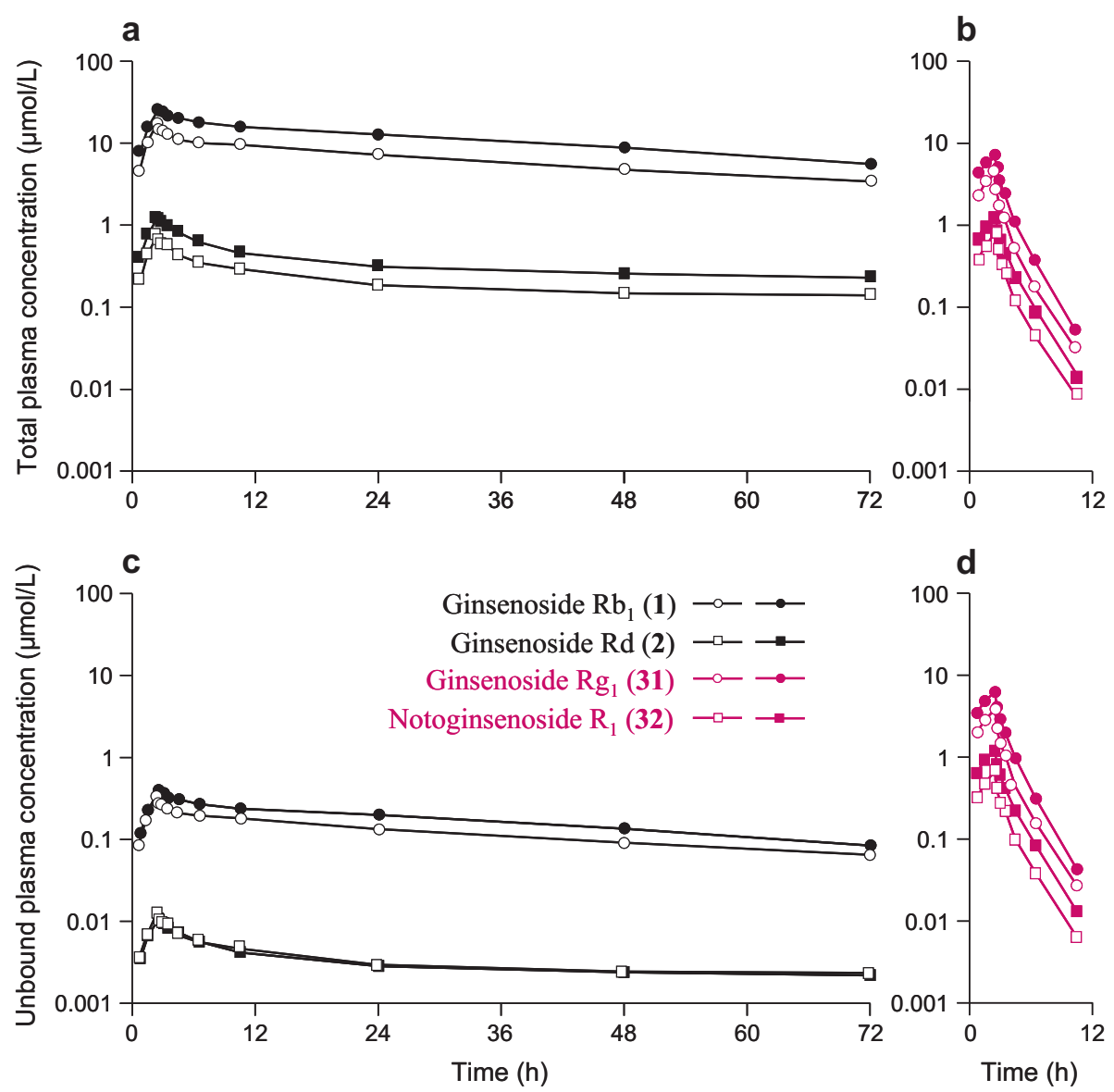

Fig. 3 Mean total (bound + unbound; a, b) and mean unbound (c, d) plasma concentrations of ginsenosides $\mathrm{Rb}_{1}(\mathbf{1}), \mathrm{Rd}^{(2)}$, and $\mathrm{Rg} \mathbf{1}_{1}(\mathbf{3 1})$ and notoginsenoside $R_{1}(32)$ over time after a single 2.5 -h intravenous infusion of XueShuanTong at $250 \mathrm{mg} /$ person in human subjects ( $\mathrm{m} 1-\mathrm{m} 8$ and f1-f6; open circle and square) and 500 mg/person in human subjects (m9-m16 and f7-f12; solid circle and square)

were mainly associated with intercompound differences in total plasma clearance $\left(\mathrm{CL}_{\text {tot, }}\right)$. Such differences in apparent volumes of distribution at steady state $\left(V_{\mathrm{SS}}\right)$ were smaller than those in $\mathrm{CL}_{\text {tot, }}$. The $V_{\mathrm{SS}}$ values of the ppd-type ginsenosides (1 and $\left.\mathbf{2}\right)$ were smaller than those of the ppt-type ginsenosides' (31 and 32); with the $V_{S S}$ of the former being smaller than the human extracellular volume $(260 \mathrm{~mL} / \mathrm{kg}$ [30]) and those of the latter approximating $260 \mathrm{~mL} / \mathrm{kg}$. The ppd-type ginsenosides (1 and 2) notably accumulated during repeated doses of XueShuanTong for 15 consecutive days, but ppt-type ginsenosides (31 and 32) did not. The accumulative factors $(R)$ of ginsenosides ( $\mathbf{1}$ and $\mathbf{2}$ ), measured in human study 2, were 5.1 and 8.0 , respectively; this measured intercompound difference in $R$ was consistent with that estimated using the elimination rate constant $k\left(0.693 / t_{1 / 2}\right)$.

Intravenous XueShuanTong or its major circulating ginsenosides exhibiting no modulation of CYP3A-mediated midazolam 1'hydroxylation

All human subjects metabolized midazolam extensively to $1^{\prime \prime}$ hydroxymidazolam on days 1 (receiving no XueShuanTong; baseline), 4 (receiving the first daily dose of XueShuanTong), and 18 (receiving the 15th daily dose of XueShuanTong). Figure 4 shows plasma concentrations of midazolam and 1'-hydroxymidazolam over time after oral dosing of midazolam tablet on these 3 days; the compounds' systemic exposure data are summarized in Supplementary Table S3. No statistically significant difference between days 1 and 4 was noted in $C_{\text {max }}, \mathrm{AUC}_{0-8 \mathrm{~h}}, \mathrm{AUC}_{0-\infty}$ or $t_{1 / 2}$ values of midazolam or in those of $1^{\prime}$-hydroxymidazolam, suggesting that there was no obvious inhibition or activation of
CYP3A, mediating midazolam 1'-hydroxylation, by intravenously administered XueShuanTong. Additionally, such differences were not detected between days 1 and 18, suggesting that there was no induction of CYP3A during the repeated doses of XueShuanTong.

Ginsenosides $R b_{1}(\mathbf{1}), R d(2)$, and $R_{1}(31)$, notoginsenoside $R_{1}$ (32), and XueShuanTong at their test concentrations did not significantly decrease the mitochondrial activity of human hepatocytes. The positive control rifampin $(20 \mu \mathrm{mol} / \mathrm{L})$, a known inducer of CYP3A, exhibited average ratios of enzyme activity (as indicated by the formation of $1^{\prime}$-hydroxymidazolam) between the treated cells and the negative control, i.e., 4.1-44.6. As shown in Fig. 5, ginsenosides $R b_{1}(\mathbf{1}), R d(2)$, and $R g_{1}(31)$ and notoginsenoside $R_{1}$ (32) exhibited such ratios of 0.4-1.9 at all test concentrations, except for notoginsenoside $R_{1}(32)$ at $100 \mu \mathrm{mol} / \mathrm{L}$ exhibiting a ratio of 2.4 for the donor HVN. This ratio of notoginsenoside $R_{1}$ (32) was only $5.5 \%(<20 \%)$ of the positive control's ratio for HVN. At the test concentrations, XueShuanTong exhibited ratios of enzyme activity ranging from 1.1 to 1.5 for the donors XSM and IZT. Such ratios for the donor HVN were 2.6-2.9, which were only $5.9 \%-6.6 \%$ of the positive control's ratio for HVN. These ginsenosides (1, 2, 31, and 32; each at $100 \mu \mathrm{mol} / \mathrm{L})$, as well as XueShuanTong (at the marker concentration $100 \mu \mathrm{mol} / \mathrm{L}$ of ginsenoside $\mathrm{Rg}_{1}$ (31) present), did not exhibit $>50 \%$ inhibition of CYP3A4 or CYP3A5 in vitro (Supplementary Table S4). To assess influence of treatment with the ginsenosides $(\mathbf{1}, \mathbf{2}, \mathbf{3 1}$, and 32) and XueShuanTong on cellular CYP3A4 and CYP3A5 mRNA expression, real-time PCR analysis was performed, and the results are shown in Fig. 5. Hepatocytes treated with rifampin resulted in notable 

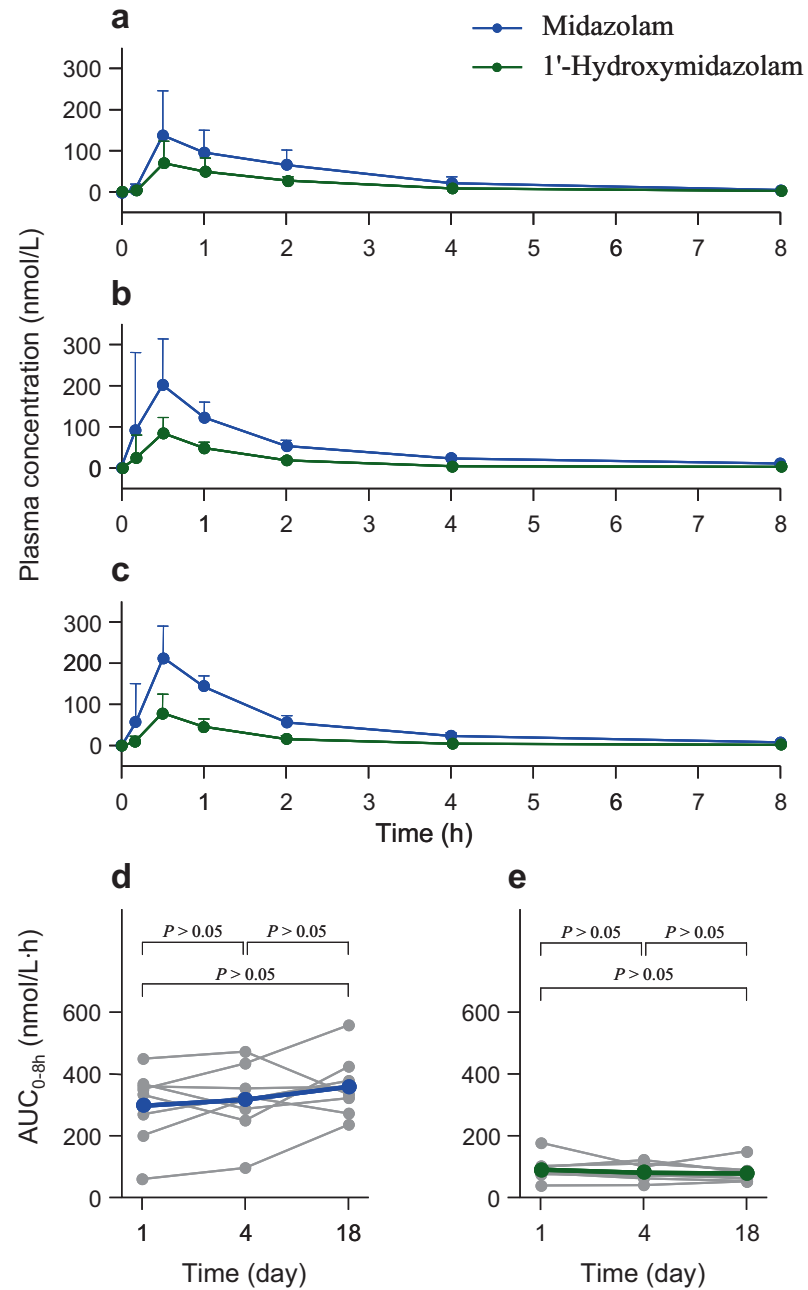

Fig. 4 Mean plasma concentrations of midazolam (blue solid circle) and its metabolite $1^{\prime}$-hydroxymidazolam (green solid circle) over time after a single oral dose of midazolam at $7.5 \mathrm{mg}$ alone (day 1, a) in human subjects (m17-m24) and with repeated doses of XueShuanTong $(500 \mathrm{mg}$ once daily via 2.5 -h intravenous infusion, from day 4 to day 18 ) on day 4 (b) and day 18 (c). Plasma $A U C_{0-8 h}$ values of midazolam (d) on day 1 , day 4 , and day 18 are compared with one another (blue solid circles for the mean values; gray solid circles for the data of individual subjects); those of $1^{\prime}$-hydroxymidazolam (e) are also similarly compared (green solid circles for the mean values; gray solid circles for the data of individual subjects). $P>0.05$ was not considered statistically significant

increases in the mRNA expression of CYP3A4 and CYP3A5, with the ratios of mRNA levels between treated and untreated cells 30 149 and 14-26, respectively. At all test concentrations, ginsenosides $\mathrm{Rb}_{1}(\mathbf{1})$, $\mathrm{Rd}(\mathbf{2})$, and $R g_{1}(\mathbf{3 1})$, notoginsenoside $\mathrm{R}_{1}(\mathbf{3 2})$, and XueShuanTong exhibited such ratios of $0.5-4.1$ and $0.5-2.6$, respectively. Collectively, intravenously administered XueShuanTong did not induce CYP3A; consistently, its major circulating ginsenosides $\mathrm{Rb}_{1}(\mathbf{1}), \mathrm{Rd}(\mathbf{2})$, or $\mathrm{Rg}_{1}$ (31) or notoginsenoside $\mathrm{R}_{1}(\mathbf{3 2})$ did not induce the enzymes.

Potential for intravenously administered XueShuanTong-drug interactions via inhibiting OATP1B by ginsenosides Given that major circulating herbal compounds are considerably bioavailable for potential XueShuanTong-drug interactions on OATP1B, the interaction study focused on ginsenosides $\mathrm{Rb}_{1}(\mathbf{1})$, Rd (2), and $\mathrm{Rg}_{1}$ (31) and notoginsenoside $\mathrm{R}_{1}$ (32). Combination of these four ginsenosides yielded additive, rather than synergistic or antagonist, inhibition of OATP1B3 and OATP1B1, as indicated by the combination indices shown in Table 2. The comparative \% inhibition of ginsenoside $\mathrm{Rb}_{1}$ (1) alone and in the presence of ginsenosides $R d(2)$ and $R_{1}$ (31) and notoginsenoside $R_{1}(\mathbf{3 2})$, at the clinically relevant dose, is shown in Supplementary Fig. S2. Adding ginsenosides $\mathrm{Rd}(\mathbf{2})$ and $\mathrm{Rg}_{1}(\mathbf{3 1})$ and notoginsenoside $\mathrm{R}_{1}$ (32) to ginsenoside $\mathrm{Rb}_{1}$ (1) significantly increased the total \% inhibition compared with the $\%$ inhibition by ginsenoside $\mathrm{Rb}_{1}(\mathbf{1})$ alone $(P<0.05)$. Therefore, the sum of estimated individual DDI indices of these four ginsenosides was used to reflect the potential for XueShuanTong-drug interactions on the transporters (Fig. 6). The ginsenosides selectively inhibited OATP1B3; the estimated total DDI indices for OATP1B3 at $C_{\max }$ for a single dose of XueShuanTong and for repeated doses of the injection on day 18 were 5.5-times and 6.3-times, respectively, as much as those for OATP1B1. The total DDI index on OATP1B3 for the repeated doses on day 18 was 0.75 . The contribution of an individual ginsenoside to the potential interaction was estimated using the percentage of its DDI index in the total index of the four ginsenosides. At $T_{\max }$ ginsenoside $\mathrm{Rb}_{1}$ (1) was the main contributor to the potential OATP1B3-mediated interaction with the percentages $79 \%$ for a single dose of XueShuanTong and $82 \%$ for repeated doses of the injection on day 18; such percentages of ginsenosides $\mathrm{Rd}(2) / \mathrm{Rg}_{1}$ (31)/notoginsenoside $\mathrm{R}_{1}$ (32) were only $4 \% / 14 \% / 3 \%$ and $7 \% / 10 \% /$ $1 \%$, respectively. Regarding the potential interaction on OATP1B1, the percentages of ginsenoside $\mathrm{Rb}_{1}$ (1) were $48 \%$ and $56 \%$, respectively; those of ginsenosides $\mathrm{Rd}(\mathbf{2}) / \mathrm{Rg}_{1}(\mathbf{3 1}) /$ notoginsenoside $\mathrm{R}_{1}$ (32) were $7 \% / 34 \% / 11 \%$ and $11 \% / 27 \% / 6 \%$, respectively. After terminating the infusion, contributions of ppt-type ginsenoside $R_{1}(31)$ and notoginsenoside $R_{1}(32)$ were reduced quickly (due to their short $t_{1 / 2}$ ). However, the total DDI index slowly decreased, with such indices for OATP1B3 at 24 and $48 \mathrm{~h}$ after starting infusion being $40 \%$ and $28 \%$, respectively, of that at $T_{\max }$ $(2.5 \mathrm{~h})$ for a single dose of the injection and $70 \%$ and $49 \%$, respectively, of that at $2.5 \mathrm{~h}$ for repeated doses of XueShuanTong on day 18 . The percentage values for OATP1B1 were $26 \%, 18 \%$, $52 \%$, and $36 \%$, respectively.

\section{DISCUSSION}

To our knowledge, this is the first human pharmacokinetic investigation of multiple ginsenosides from intravenously administered XueShuanTong, prepared from a standardized extract of $P$. notoginseng roots (Sanqi), and it serves as the first step in determining the potential for XueShuanTong-drug interactions. Based on this investigation, unchanged (rather than metabolized) ginsenosides $\mathrm{Rb}_{1}(\mathbf{1})$, $\mathrm{Rd}(\mathbf{2})$, and $\mathrm{Rg}_{1}(\mathbf{3 1})$ and notoginsenoside $\mathrm{R}_{1}$ (32) are the major circulating XueShuanTong compounds, which are considerably bioavailable for drug interactions related to the herbal medicine. This is different from the situation after oral dosing of Sanqi extract [22, 31]. Ginsenosides are poorly absorbed from the gastrointestinal tract due to their poor membrane permeability; the unabsorbed ginsenosides can be deglycosylated by the human gut microbiota followed by further oxidation of the deglycosylated metabolites. Although several combinatorial metabolites represent the major circulating forms of ginsenosides for orally dosed Sanqi extract, exposure levels of these metabolites are notably different among human individuals (relative standard deviations of $C_{\max }$ and AUC, 51\%-150\% and 54\%-168\%, respectively) [22]. After intravenous infusion of XueShuanTong, such interindividual differences for the major circulating ginsenosides $(\mathbf{1}, \mathbf{2}, \mathbf{3 1}$, and $\mathbf{3 2})$ are relatively small, as indicated by relative standard deviations of $11 \%-23 \%$ (for $C_{\max }$ ) and $13 \%-33 \%$ (for AUC). Substantial intercompound differences in systemic exposure to XueShuanTong ginsenosides are attributable mainly to their great differences in compound dose level, i.e., over three orders of magnitude. Unlike other ppt-type ginsenosides (31 and 32; compound dose level, $>10 \mu \mathrm{mol} /$ day), ginsenoside $\operatorname{Re}(\mathbf{3 3})$ has a 

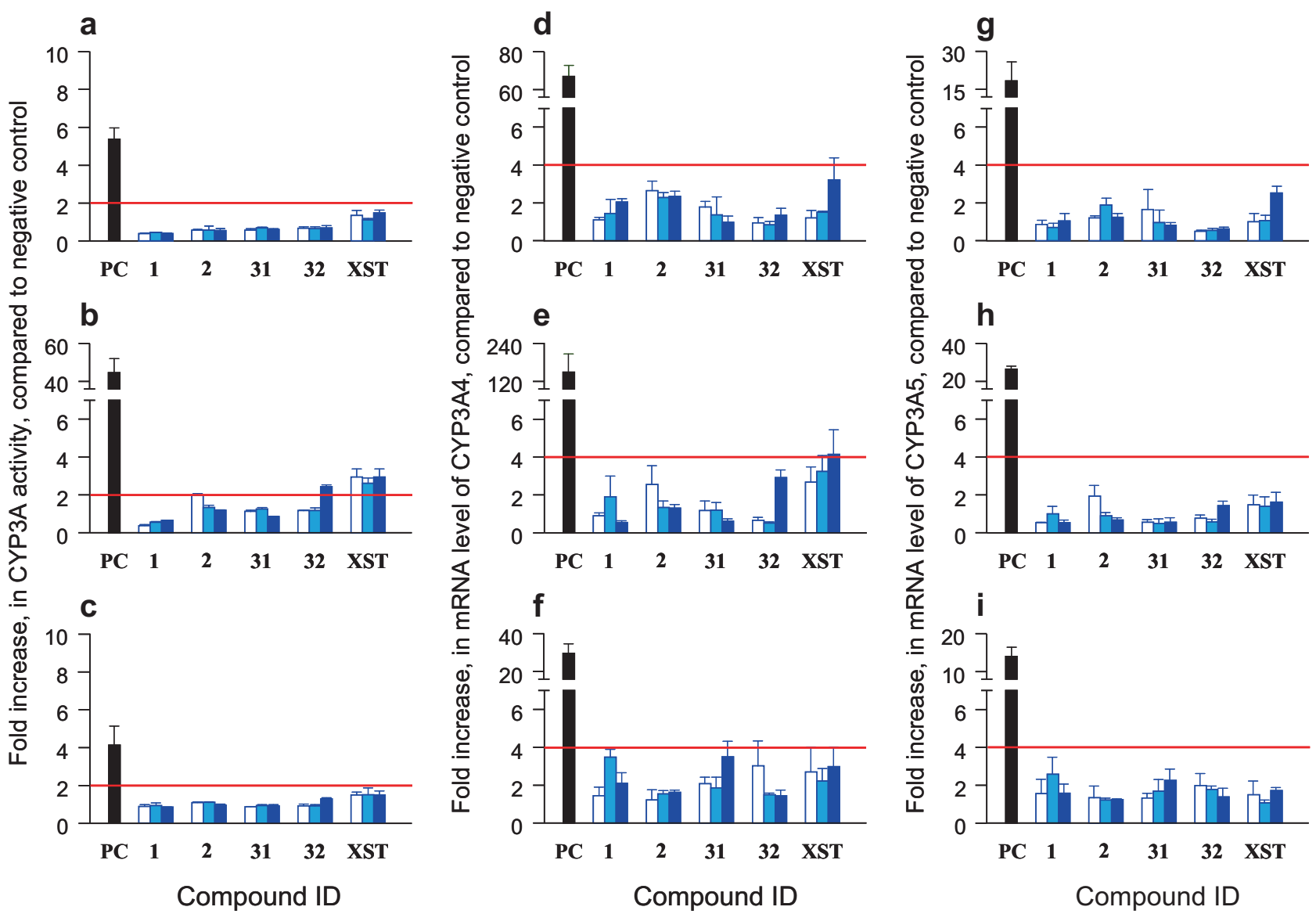

Fig. 5 Inductive effects of ginsenosides $\mathrm{Rb}_{1}(\mathbf{1})$, $\mathrm{Rd}(\mathbf{2})$, and $\mathrm{Rg}_{1}(\mathbf{3 1})$, notoginsenoside $\mathrm{R}_{1}(\mathbf{3 2})$, and XueShuanTong (XST) on CYP3A activity (ac), CYP3A4 mRNA (d-f), and CYP3A5 mRNA (g-i). These ginsenosides (1, 2, 31, and 32) and XueShuanTong (XST) were tested at low, intermediate, and high concentrations (open, light blue, and blue bars, respectively), i.e., 1/10/100 $\mu \mathrm{mol} / \mathrm{L}$, except for ginsenoside Rd (2) at $0.1 /$ $1 / 10 \mu \mathrm{mol} / \mathrm{L}$ and XueShuanTong (XST) at the marker concentrations $1 / 10 / 100 \mu \mathrm{mol} / \mathrm{L}$ of ginsenoside Rg ${ }_{1}$ (31) present. Cryopreserved human hepatocytes from three donors [XSM $(\mathbf{a}, \mathbf{d}, \mathbf{g}), \operatorname{HVN}(\mathbf{b}, \mathbf{e}, \mathbf{h})$, and IZT $(\mathbf{c}, \mathbf{f}, \mathbf{i})]$ were used; rifampin $(20 \mu \mathrm{mol} / \mathrm{L})$, a known inducer of CYP3A, served as a positive control (PC)

low $C_{\max }$ probably due to its greater $\mathrm{CL}_{\text {tot, }}$. In addition to $C_{\max }$ plasma protein binding $\left(f_{\mathrm{u}}\right)$ and terminal half-life $\left(t_{1 / 2}\right)$ also govern the contribution of an individual ginsenoside to potential XueShuanTong-drug interactions. In addition to intercompound differences in $f_{\mathrm{u}}$ (particularly between the ppd-type and ppt-type ginsenosides), interindividual differences in $f_{\mathrm{u}}$ of the highly protein-bound ppd-type ginsenosides (1 and 2 ) should also be considered in predicting the clinical outcome of the potential interactions; this requires developing more accurate and precise assays for such highly bound compounds. It is worth mentioning that ppd-type ginsenoside $\mathrm{Rb}_{1}(\mathbf{1})$ exhibits notably longer $t_{1 / 2}$ in humans than in rats, i.e., 42.1 and $17.1 \mathrm{~h}$, respectively (Supplementary Table S5). These interspecies differences, as well as the related interindividual differences in humans, should also be considered in predicting the clinical outcome of the potential interactions. Ginsenoside Rd (2) circulating after intravenous dosing of XueShuanTong exhibits notably longer $t_{1 / 2}(58-307 \mathrm{~h})$ than that after intravenous dosing of purified ginsenoside Rd $(\sim 18 \mathrm{~h})$ [32]. This discrepancy most likely results from a pharmacokinetic matrix effect of XueShuanTong, which contains both ginsenosides $\mathrm{Rb}_{1}(\mathbf{1})$ and $\mathrm{Rd}(\mathbf{2})$. Our earlier study suggests that ginsenoside $\mathrm{Rb}_{1}$ can be transformed into ginsenoside $\mathrm{Rd}$ by human hepatic cytosolic glucosidase [12]. During repeated doses of XueShuanTong, such deglycosylation of ginsenoside $\mathrm{Rb}_{1}(\mathbf{1})$ can enhance the accumulation of ginsenoside $\mathrm{Rd}(\mathbf{2})$ in the systemic circulation.
Prior studies of the potential for ginseng-drug interactions involve assessing the modulation of human cytochrome P450 enzymes (P450s), UDP-glucuronosyltransferases (UGTs), P- glycoprotein (P-gp), and OATP1Bs by ginsenosides [11, 12, 33-37]. It is clear that bulky (extensively glycosylated and highly polar) ginsenosides, such as ginsenosides $R b_{1}, R b_{2}, R c, R d, R e$, and $R g_{1}$, exhibit weak inhibition of P450s, UGTs, and P-gp in vitro, with $\mathrm{IC}_{50}>50$ $\mu \mathrm{mol} / \mathrm{L}$. Based on our current human pharmacokinetic investigation of XueShuanTong, the in vitro concentrations necessary to inhibit these enzymes and transporter were not realized in vivo at its clinically relevant dose. Deglycosylated metabolites of ginsenosides [compound-K, 20(S)-protopanaxadiol, and 20(S)-protopanaxatriol] and ginsenoside $\mathrm{Rg}_{3}$ exhibit relatively potent inhibition of the enzymes and transporter, but these compounds were poorly or negligibly detected in humans after dosing XueShuanTong. Accordingly, the potential for XueShuanTong-drug interactions via inhibiting P450s, UGTs, or P-gp was not considered in this investigation. Due to the clinical study by Malati et al. [20]. and the substantially higher systemic exposure to ginsenosides after intravenous administration than after oral administration, the potential of XueShuanTong to induce CYP3A was assessed in this investigation. Both the human study and the in vitro induction study demonstrate that repeatedly dosing intravenous XueShuanTong, at the label dose $500 \mathrm{mg} /$ day, does not induce human CYP3A. Consistent with our current in vitro data, other investigators also reported no cellular induction of human CYP3A by 


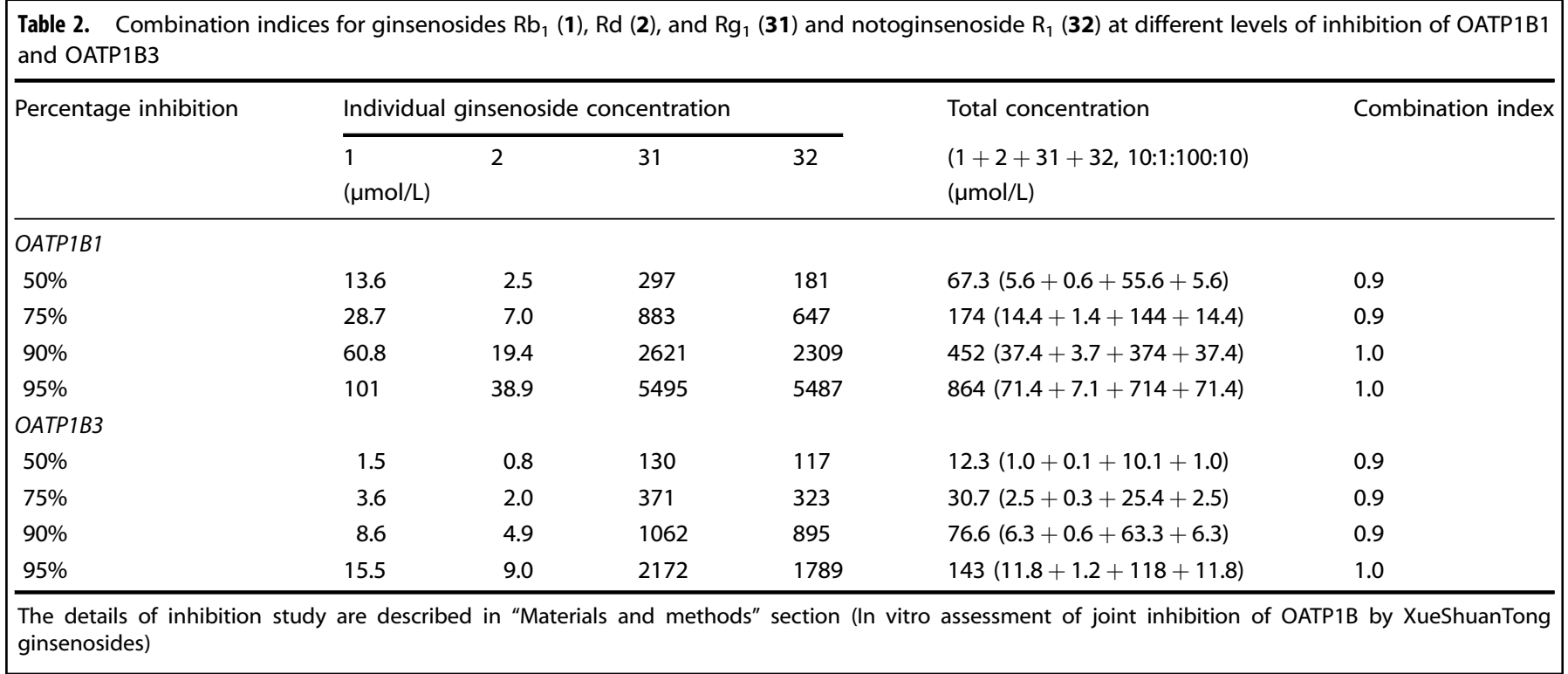

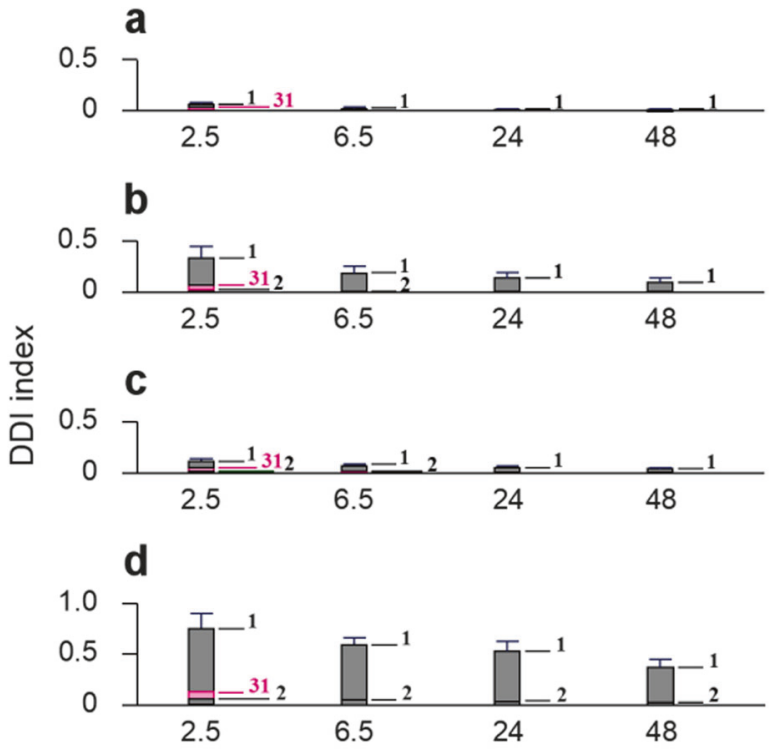

Time after starting infusion of XueShuanTong $(\mathrm{h})$

Fig. 6 Estimated total DDI indices on OATP1B1 $(\mathbf{a}, \mathbf{c})$ and OATP1B3 (b, d), for a single 2.5-h intravenous infusion of XueShuanTong (the label dose $500 \mathrm{mg} ; \mathbf{a}, \mathbf{b}$ ) and for repeated doses of XueShuanTong (500 mg, once daily) on day 18 (c, d). 1, ginsenoside $\mathrm{Rb}_{1} ; \mathbf{2}$, ginsenoside $\mathrm{Rd} ; \mathbf{3 1}$, ginsenoside $\mathrm{Rg}_{1}$; and 32 , notoginsenoside $\mathrm{R}_{1}$

ginsenosides $\mathrm{Rb}_{1}, \mathrm{Rd}$, or $\mathrm{Rg}_{1}[38,39]$. Notably, our conclusion (i.e., intravenous XueShuanTong not inducing human CYP3A) should not be extrapolated simply to oral ginsenoside-containing products due to notable differences in human exposure to ginsenosides between the two routes of administration. The recently reported impact of gut microbiota on hepatic expression of P450s in mice $[40,41]$ suggests that interactions of ginsenosides with the gut microbiota should be considered in assessing the potential of oral ginsenoside-containing products to induce CYP3A. In addition, XueShuanTong appears not to induce CYP1A2 and CYP2B6 (Supplementary Fig. S3).

The potential for XueShuanTong-drug interactions on OATP1Bs was predicted, based on understanding of bioavailability and pharmacokinetics of XueShuanTong ginsenosides in humans and their inhibitory potencies on the human transporters. Because the ginsenosides selectively inhibit OATP1B3, XueShuanTong is most likely to alter pharmacokinetics of selective substrates of OATP1B3 when coadministered, rather than selective substrates of OATP1B1 or dual substrates of OATP1B1/1B3. Several selective OATP1B3 substrates, such as cholecystokinin-8, telmisartan, amanitin, dioscin, convallatoxin, ouabain, dihydroouabain, ouabagenin, and ppt-type ginsenosides, have been reported [11, 12, 4246]. Among the circulating ginsenosides, ppd-type ginsenoside $\mathrm{Rb}_{1}$ (1) predominantly contributes to the potential OATP1B3mediated XueShuanTong-drug interaction, whereas the other circulating ginsenosides $\mathrm{Rd}(\mathbf{2})$ and $\mathrm{Rg}_{1}(\mathbf{3 1})$ and notoginsenoside $R_{1}$ (32) are minor contributors to the potential interaction; all these four compounds inhibit the transporter in an additive manner. For OATP1B3-mediated interactions, the compoundcontribution profile of XueShuanTong (derived from P. notoginseng roots) is different from that of ShenMai (a steamed $P$. ginseng root-containing injection for intravenous administration), i.e., contributions of multiple ShenMai ginsenosides of ppd-type and other types are comparable [12]. Due to the long $t_{1 / 2}$ of ppd-type ginsenosides $\mathrm{Rb}_{1}(\mathbf{1})$ and $\mathrm{Rd}(\mathbf{2})$ in humans, the mean total DDI index for the potential XueShuanTong-drug interaction slowly decreases after terminating the infusion (as indicated by the value at $24 \mathrm{~h}$ after starting the infusion being $>40 \%$ of that at $T_{\text {max }}$ ). Additionally, this index can steadily increase during repeated doses of XueShuanTong (as indicated by the mean total DDI indices on day 18 after repeated doses being $\sim 2.5$ times as much as the associated mean values for the first daily dose; e.g., at $2.5 \mathrm{~h}$ after starting infusion, increasing from 0.3 to 0.8 ). In addition to the mean total DDI index, the associated interindividual difference should also be considered in interpreting the study results; for the potential OATP1B3-mediated XueShuanTong-drug interaction, even while receiving the same doses, some individuals can be at higher risk of interaction than others. Such variation may result from interindividual differences in exposure to the circulating ginsenosides and in their in vivo $I_{50}$ values for OATP1B3. The exposure differences involve interindividual differences not only in $C_{\max }$ and $f_{\mathrm{u}}$ of the ginsenosides but also in their $t_{1 / 2}$, mainly for long-circulating and extensively plasma-protein-bound ginsenosides $\mathrm{Rb}_{1}(\mathbf{1})$ and $\mathrm{Rd}(\mathbf{2})$. Based on such exposure differences of the ginsenosides, the estimated highest individual subject value of the total DDI index for the potential XueShuanTong-drug interaction may be twice as much as the estimated mean value of this total 
DDI index for all the subjects. However, this does not indicate the exact interindividual differences in the total DDI index, because the interindividual in vivo $\mathrm{IC}_{50}$ differences are not incorporated; currently, it is technically difficult to assess the in vivo $I C_{50}$.

Increased awareness of drug interaction potential of herbal medicines will help ensure the safe and effective use of both herbal medicines and coadministered synthetic drugs. Information on the potential for interactions should be an important part of the package leaflet of herbal medicines. However, most Chinese herbal medicines currently lack such information, including the extensively used XueShuanTong. To this end, we determined the potential for XueShuanTong-drug interactions. In summary, ginsenosides $\mathrm{Rb}_{1}$ (1), $\mathrm{Rd}(\mathbf{2})$, and $\mathrm{Rg}_{1}$ (31) and notoginsenoside $\mathrm{R}_{1}$ (32) are the key XueShuanTong compounds that are considerably bioavailable for drug interactions; their interactionrelated human pharmacokinetic profiles consist of compound dose-dependent levels of systemic exposure and, for ginsenosides $\mathrm{Rb}_{1}(\mathbf{1})$ and $\mathrm{Rd}(\mathbf{2})$, long terminal half-lives and extensive binding to plasma proteins. Repeatedly dosing intravenous XueShuanTong does not induce human CYP3A4/3A5. Based on human pharmacokinetics and overall inhibitory potency of its bioavailable ginsenosides, intravenously dosed XueShuanTong is found to have high potential for OATP1B3-mediated drug interactions, which is attributed chiefly to ginsenoside $\mathrm{Rb}_{1}(\mathbf{1})$. This finding informs the need for further model-based determination of the interaction potential for XueShuanTong and, if necessary, associated clinical drug interaction study. Based on the results of our current investigation, caution is advised when (1) increasing the dose and/or the infusion rate of XueShuanTong, (2) coadministering XueShuanTong with drugs having their disposition/elimination largely dependent on OATP1B3 and having narrow therapeutic indices, and (3) using XueShuanTong in patients with hypoalbuminemia and/or impaired liver function and in elderly patients receiving polypharmacy. To this end, pharmacokinetic investigation of XueShuanTong in patients and identification of markers that can indicate individual exposure to or in vivo OATP1B3 IC 50 of the ginsenosides are also warranted to provide information necessary to guide clinical decision making.

\section{ACKNOWLEDGEMENTS}

This work was supported in part by the National Key R\&D Program of China (Grant 2018YFC1704500); by the National Science and Technology Major Project of China "Key New Drug Creation and Manufacturing Program" (Grants 2012ZX09101201-005 and 2017ZX09301012-006); by the National Natural Science Foundation of China (Grant 81673582); by the Strategic Priority Research Program of the Chinese Academy of Sciences (Grant XDA12050306); and by the National Basic Research Program of China (Grant 2012CB518403).

\section{AUTHOR CONTRIBUTIONS}

Participated in the research design: $C L, J L Y, W N, Y H H, S P$. Conducted experiments: JLY, SP, WN, XND, OEO, FQW, YFL. Performed the data analysis: CL, JLY, SP, WN. Wrote or contributed to the writing of the manuscript: $C L, S P, J L Y$.

\section{ADDITIONAL INFORMATION}

The online version of this article (https://doi.org/10.1038/s41401-019-0273-1) contains supplementary material, which is available to authorized users.

Conflict of interest: The authors declare that they have no conflict of interest.

\section{REFERENCES}

1. Bailey DG, Spence JD, Munoz C, Arnold JMO. Interaction of citrus juices with felodipine and nifedipine. Lancet. 1991;37:268-9.

2. Bailey DG, Arnold JMO, Bend JR, Tran LT, Spence JD. Grapefruit juice-felodipine interaction: reproducibility and characterization with the extended release drug formulation. Br J Clin Pharmacol. 1995;40:135-40.
3. Ruschitzka F, Meier PJ, Turina M, Luscher TF, Noll G. Acute heart transplant rejection due to Saint John's wort. Lancet. 2000;355:548-9.

4. Breidenbach T, Kliem V, Burg M, Radermacher J, Hoffmann MW, Klempnauer J. Profound drop of cyclosporine a whole blood trough levels caused by St. John's wort (Hypericum perforatum). Transplantation. 2000;69:2229-30.

5. Barone GW, Gurley BJ, Ketel BL, Lightfoot ML, Abul-Ezz SR. Drug interaction between St. John's wort and cyclosporine. Ann Pharm. 2000;34:1013-6.

6. Johnson EJ, Gonzalez-Perez V, Tian D-D, Lin Y-S, Unadkat JD, Rettie AE, et al. Selection of priority natural products for evaluation as potential precipitants of natural product-drug interactions: a NaPDI center recommended approach. Drug Metab Dispos. 2018;46:1046-52.

7. Komoroski BJ, Zhang SM, Cai HB, Hutzler M, Frye R, Tracy TS, et al. Induction and inhibition of cytochromes P450 by the St. John's wort constituent hyperforin in human hepatocyte cultures. Drug Metab Dispos. 2004;32:512-8.

8. Qiu FR, Zhang R, Sun JG, Jiye A, Hao HP, Peng Y, et al. Inhibitory effects of seven components of danshen extract on catalytic activity of cytochrome P450 enzyme in human liver microsomes. Drug Metab Dispos. 2008;36:1308-14.

9. Gurley BJ, Fifer EK, Gardner Z. Pharmacokinetic herb-drug interactions (part 2): drug interactions involving popular botanical dietary supplements and their clinical relevance. Planta Med. 2012;78:1490-514.

10. Gufford BT, Chen G, Lazarus P, Graf TN, Oberlies NH, Paine MF. Identification of diet-derived constituents as potent inhibitors of intestinal glucuronidation. Drug Metab Dispos. 2014;42:1675-83.

11. Jiang RR, Dong JJ, Li XX, Du FF, Jia WW, Xu F, et al. Molecular mechanisms governing different pharmacokinetics of ginsenosides and potential for ginsenoside-perpetrated herb-drug interactions on OATP1B3. $\mathrm{Br} J$ Pharmacol. 2015;172:1059-73.

12. Olaleye OE, Niu W, Du FF, Wang FQ, Xu F, Pintusophon S, et al. Multiple circulating saponins from intravenous ShenMai inhibit OATP1Bs in vitro: potential joint precipitants of drug interactions. Acta Pharmacol Sin. 2019;40:833-49.

13. Fugh-Berman A. Herb-drug interactions. Lancet. 2000;355:134-8.

14. Sparreboom A, Cox MC, Acharya MR, Figg WD. Herbal remedies in the United States: potential adverse interactions with anticancer agents. J Clin Oncol. 2004;22:2489-503.

15. Tachjian A, Maria V, Jahangir A. Use of herbal products and potential interactions in patients with cardiovascular diseases. J Am Coll Cardiol. 2010;55: 515-25.

16. Yu K, Chen F, Li C. Absorption and disposition of saponins: what do we know and what do we need to know? Curr Drug Metab. 2012;13:577-98.

17. Hermann R, von Richter O. Clinical evidence of herbal drugs as perpetrators of pharmacokinetic drug interactions. Planta Med. 2012;78:1458-77.

18. Ramanathan MR, Penzak SR. Pharmacokinetic drug interactions with Panax ginseng. Eur J Drug Metab Pharmacokinet. 2017;42:545-57.

19. Awortwe C, Makiwane M, Reuter H, Muller C, Louw J, Rosenkranz B. Critical evaluation of causality assessment of herb-drug interactions in patients. Br J Clin Pharmacol. 2018;84:679-93.

20. Malati CY, Robertson SM, Hunt JD, Chairez C, Alfaro RM, Kovacs JA, et al. Influence of Panax ginseng on cytochrome P450 (CYP)3A and P-glycoprotein (P-gp) activity in healthy participants. J Clin Pharmacol. 2012;52:932-9.

21. Gurley BJ, Gardner SF, Hubbard MA, Williams DK, Gentry WB, Cui YY, et al. Clinical assessment of effects of botanical supplementation on cytochrome P450 phenotypes in the elderly. Drug Aging 2005;22:525-39.

22. Hu ZY, Yang JL, Cheng $C$, Huang YH, Du FF, Wang FQ, et al. Combinatorial metabolism notably affects human systemic exposure to ginsenosides from orally administered extract of Panax notoginseng roots (Sanqi). Drug Metab Dispos. 2013;41:1457-69.

23. Fu XX, Xiao WJ, Lu J, Wu LW, Yang F. Retrospective analysis of thrombolysis therapy for 64 cases of acute myocardial infarction with elevated ST segment. Chin J Integr Med. 2009;15:462-5.

24. Gui QF, Yang YM, Ying SH, Zhang MM. XueShuanTong improves cerebral blood perfusion in elderly patients with lacunar infarction. Neural Regen Res. 2013:8:792-801.

25. Zhang R, Zhang GF, Chen J. Effects of XueShuanTong injection on inflammatory cytokines, homocysteine and prognosis in patients with acute cerebral infarction. J Hubei Univ Chin Med. 2016;18:24-7.

26. Guo B, Li C, Wang GJ, Chen LS. Rapid and direct measurement of free concentrations of highly protein-bound fluoxetine and its metabolite norfluoxetine in plasma. Rapid Commun Mass Spectrom. 2006;20:39-47.

27. Fahmi OA, Kish M, Boldt S, Obach RS. Cytochrome P450 3A4 mRNA is a more reliable marker than CYP3A4 activity for detecting pregnane $X$ receptoractivated induction of drug-metabolizing enzymes. Drug Metab Dispos. 2010; 38:1605-11.

28. Livak KJ, Schmittgen TD. Analysis of relative gene expression data using real-time quantitative PCR and the $2^{-\Delta \Delta C_{T}}$ method. Methods. 2001;25:402-8. 
29. Chou TC. Drug combination studies and their synergy quantification using the Chou-Talalay method. Cancer Res. 2010;70:440-6.

30. Davies B, Morris T. Physiological parameters in laboratory animals and humans. Pharm Res. 1993;10:1093-5.

31. Liu HF, Yang JL, Du FF, Gao XM, Ma XT, Huang YH, et al. Absorption and disposition of ginsenosides after oral administration of Panax notoginseng extract to rats. Drug Metab Dispos. 2009;37:2290-8.

32. Zeng $X$, Deng $Y H$, Feng $Y$, Liu YM, Yang L, Huang Y, et al. Pharmacokinetics and safety of ginsenoside $\mathrm{Rd}$ following a single or multiple intravenous dose in healthy Chinese volunteers. J Clin Pharmacol. 2010;50:285-92.

33. Liu Y, Zhang JW, Li W, Ma H, Sun J, Deng MC, et al. Ginsenoside metabolites, rather than naturally occurring ginsenosides, lead to inhibition of human cytochrome P450 enzymes. Toxicol Sci. 2006;91:356-64.

34. Hao M, Zhao YQ, Chen PZ, Huang $H$, Liu $H$, Jiang $H L$, et al. Structure-activity relationship and substrate-dependent phenomena in effects of ginsenosides on activities of drug-metabolizing P450 enzymes. PLoS ONE. 2008;3:e2697.

35. Fang ZZ, Cao YF, Hu CM, Hong M, Sun XY, Ge GB, et al. Structure-inhibition relationship of ginsenosides towards UDP-glucuronosyltransferases (UGTs). Toxicol Appl Pharmacol. 2013;267:149-54.

36. Zhang JW, Zhou F, Wu XL, Gu Y, Ai H, Zheng YT, et al. 20(S)-ginsenoside Rh2 noncompetitively inhibits P-glycoprotein in vitro and in vivo: a case for herb-drug interactions. Drug Metab Dispos. 2010;38:2179-87.

37. Li N, Wang DD, Ge GB, Wang XL, Liu Y, Yang L. Ginsenoside metabolites inhibit Pglycoprotein in vitro and in situ using three absorption models. Planta Med. 2014;80:290-6.
38. Hao M, Ba Q, Yin J, Li JQ, Zhao YQ, Wang H. Deglycosylated ginsenosides are more potent inducers of CYP1A1, CYP1A2 and CYP3A4 expression in HepG2 cells than glycosylated ginsenosides. Drug Metab Pharmacokinet. 2011;26:201-5.

39. Seong SJ, Kang WY, Heo JK, Jo J, Choi WG, Liu KH, et al. A comprehensive in vivo and in vitro assessment of the drug interaction potential of red ginseng. Clin Ther. 2018:40:1322-37.

40. Claus SP, Ellero SL, Berger B, Krause L, Bruttin A, Molina J, et al. Colonizationinduced host-gut microbial metabolic interaction. mBio. 2011;2:e00271-10.

41. Selwyn FP, Cui JY, Klaassen CD. RNA-Seq quantification of hepatic drug processing genes in germ-free mice. Drug Metab Dispos. 2015;43:1572-80.

42. Ismair MG, Stieger B, Cattori V, Hagenbuch B, Fried M, Meier PJ, et al. Hepatic uptake of cholecystokinin octapeptide by organic anion-transporting polypeptides OATP4 and OATP8 of rat and human liver. Gastroenterology. 2001;121:1185-90.

43. Ishiguro N, Maeda K, Kishimoto W, Saito A, Harada A, Ebner T, et al. Predominant contribution of OATP1B3 to the hepatic uptake of telmisartan, an angiotensin II receptor antagonist, in humans. Drug Metab Dispos. 2006;34:1109-15.

44. Letschert K, Faulstich H, Keller D, Keppler D. Molecular characterization and inhibition of amanitin uptake into human hepatocytes. Toxicol Sci. 2006;91:140-9.

45. Zhang AJ, Wang CY, Liu Q, Meng Q, Peng JY, Sun HJ, et al. Involvement of organic anion-transporting polypeptides in the hepatic uptake of dioscin in rats and humans. Drug Metab Dispos. 2013:41:994-1003.

46. Gozalpour E, Greupink R, Wortelboer HM, Bilos A, Schreurs M, Russel FG, et al. Interaction of digitalis-like compounds with liver uptake transporters NTCP OATP1B1, and OATP1B3. Mol Pharmacol. 2014;11:1844-55. 